\title{
VEGAS-SSS. A VST early-type galaxy survey: analysis of small stellar systems
}

\section{Testing the methodology on the globular cluster system in NGC $3115^{\star}$}

\author{
Michele Cantiello ${ }^{1}$, Massimo Capaccioli ${ }^{2,3}$, Nicola Napolitano ${ }^{3}$, Aniello Grado ${ }^{3}$, Luca Limatola ${ }^{3}$, Maurizio Paolillo ${ }^{2,4}$, \\ Enrica Iodice ${ }^{3}$, Aaron J. Romanowsky ${ }^{5,6}$, Duncan A. Forbes ${ }^{7}$, Gabriella Raimondo ${ }^{1}$, Marilena Spavone ${ }^{3}$, \\ Francesco La Barbera ${ }^{3}$, Thomas H. Puzia ${ }^{8,9}$, and Pietro Schipani ${ }^{3}$
}

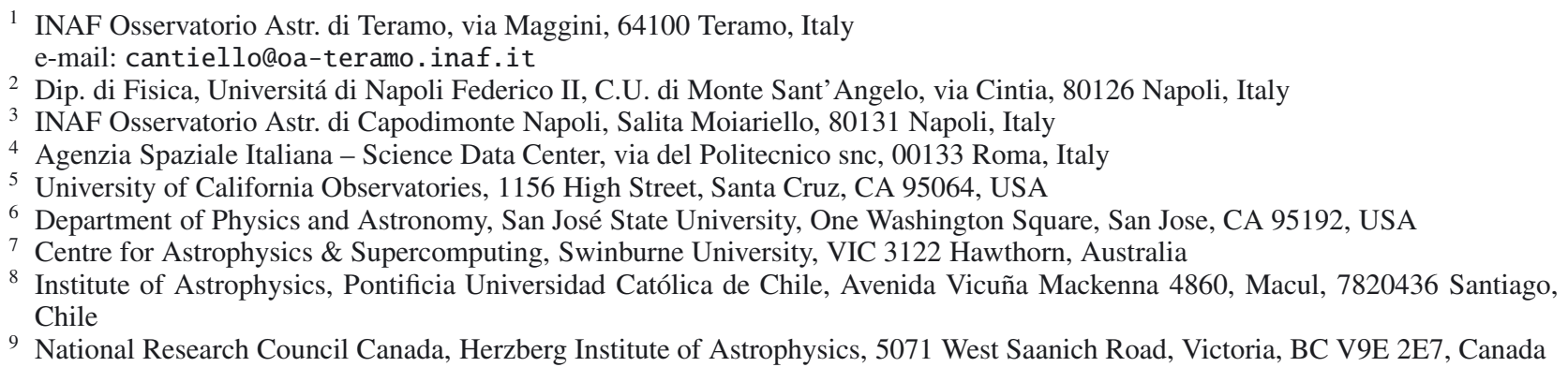

Received 16 October 2014 / Accepted 20 November 2014

\section{ABSTRACT}

\begin{abstract}
We present a study of globular clusters (GCs) and other small stellar systems (SSSs) in the field of NGC 3115, observed as part of the ongoing wide-field imaging survey VEGAS, carried out with the $2.6 \mathrm{~m}$ VST telescope. We used deep $g$ and $i$ observations of NGC 3115, a well-studied lenticular galaxy that is covered excellently well in the scientific literature. This is fundamental to test the methodologies, verify the results, and probe the capabilities of the VEGAS-SSS. Leveraging the large field of view of the VST allowed us to accurately study the distribution and properties of SSSs as a function of galactocentric distance, well beyond $\sim 20$ galaxy effective radii, in a way that is rarely possible. Our analysis of colors, magnitudes, and sizes of SSS candidates confirms the results from existing studies, some of which were carried out with 8-10 m class telescopes, and further extends them to previously unreached galactocentric distances with similar accuracy. In particular, we find a color bimodality for the GC population and a de Vaucouleurs $r^{1 / 4}$ profile for the surface density of GCs similar to the galaxy light profile. The radial color gradient of blue and red GCs previously found, for instance, by the SLUGGS survey with Subaru and Keck data, is further extended out to the largest galactocentric radii inspected, $\sim 65 \mathrm{kpc}$. In addition, the surface density profiles of blue and red GCs taken separately are well approximated by a $r^{1 / 4}$ density profile, with the fraction of blue GCs being slightly larger at larger radii. We do not find hints of a trend for the red GC subpopulation and for the GC turnover magnitude to vary with radius, but we observe a $\sim 0.2$ mag difference in the turnover magnitude of the blue and red GC subpopulations. Finally, from inspecting SSS sizes and colors, we obtain a list of ultracompact dwarf galaxies and GC candidates suitable for future spectroscopic follow-up. In conclusion, our study shows i) the reliability of the methodologies developed to study SSSs in the field of bright early-type galaxies; and ii) the great potential of the VEGAS survey to produce original results on SSSs science, mainly thanks to the wide-field imaging adopted.
\end{abstract}

Key words. galaxies: stellar content - galaxies: statistics - galaxies: individual: NGC 3115 - galaxies: star clusters: general surveys - catalogs

\section{Introduction}

Studying the properties of old star clusters in and around galaxies is one of the keystones for understanding the formation and evolution of galaxies (Ashman \& Zepf 1992; Forbes et al. 1997; Côté et al. 1998; Brodie \& Strader 2006; Tonini 2013). Because it is relatively easy to detect them out to large galactocentric distances, and because their host stellar populations are less complex than massive galaxies, star clusters are an accurate and relatively straightforward tool for unveiling the mechanisms that

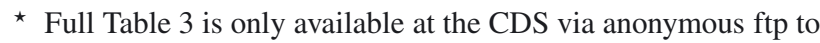
cdsarc.u-strasbg.fr (130.79.128.5) or via http://cdsarc.u-strasbg.fr/viz-bin/qcat?]/A+A/576/A14 produced the current distribution and evolutionary properties of stars in the host galaxy.

The surroundings of massive galaxies are populated by a zoo of small stellar systems (SSSs): globular clusters (GCs), extended clusters (ECs), ultracompact dwarfs (UCDs), dwarf spheroidals (dSphs), dwarf ellipticals (dEs), compact ellipticals (cE), etc. (see, e.g., Forbes et al. 2013, and references therein). The characteristic magnitude, colors and half-light radii for some SSS classes are given in Table 1. We emphasize that the distinction between the different SSS types is sometimes not trivial, and somewhat arbitrary because of the lack of a sharp distinction between the classes of SSSs, as revealed, for example, by the scaling relations of mass, radius, luminosity, 
Table 1. Typical properties of various classes of SSS.

\begin{tabular}{lcccl}
\hline \hline SSS Class & $M_{V}$ (mag) & $V-I$ & $R_{H}$ pc & Reference \\
\hline GC & -11 to $\gtrsim-5$ & $0.8-1.2$ & $2-8$ & 3,6 \\
$\mathrm{EC}^{a}$ & -6 to $<-4$ & $\sim 1.2$ & $8-50$ & $6,7,13$ \\
$\mathrm{UCD}$ & -14 to -9 & $0.7-1.3$ & $8-100$ & $1,2,4,6$ \\
$\mathrm{dE}$ & -16 to -12 & $0.8-1.2$ & $300-1000$ & 5 \\
$\mathrm{cE}$ & -18 to -15 & $\sim 1.2$ & $100-500$ & $5,11,12$ \\
$\mathrm{dSph}$ & -12 to $\gtrsim-5$ & $0.8-1.2$ & $50-1500$ & $6,8,9,10$ \\
\hline
\end{tabular}

Notes. ${ }^{(a)}$ Objects with similar luminosity and size have been also dubbed faint fuzzies (Larsen \& Brodie 2000; Peng et al. 2006; Forbes et al. 2013).

References. (1) Drinkwater et al. (2004); (2) Mieske et al. (2006); (3) Harris (1996); (4) Mieske et al. (2012); (5) Chiboucas et al. (2011); (6) Brodie et al. (2011); (7) Madrid (2011); (8) McConnachie (2012); (9) Karachentsev et al. (2001); (10) Rejkuba et al. (2006); (11) Misgeld et al. (2009); (12) Mieske et al. (2012); (13) Huxor et al. (2005).

central surface brightness, or velocity dispersion (Drinkwater et al. 2004; Misgeld \& Hilker 2011; Chiboucas et al. 2011; McConnachie 2012). A natural explanation of this lack of clear class-boundaries is that there is none. Indeed, the transformation processes occurring in dense environments may disrupt or transform massive SSSs, littering the galaxy field with the remains of disrupted system: low-mass SSSs, stellar streams, etc. (Bassino et al. 1994; West et al. 1995; D'Abrusco et al. 2013, 2014).

Characterizing the properties of the wealth of SSSs in the potential well of the host galaxy is fundamental for the understanding of their origin, and is an important tool for gauging the growth of the galaxy and, more in general, of cosmic structures.

In this context, our study is dedicated to the analysis of SSSs in NGC 3115, and is the first of a series aimed at analyzing SSSs in bright early-type galaxies, which are observed as part of the ongoing imaging VST survey VEGAS ("VST early-type galaxy survey", distributed over many semesters; GTO-INAF program, P.I. Massimo Capaccioli).

An overview of VEGAS and its scientific aims is presented in Capaccioli et al. (in prep.). At completion, the survey will have collected detailed photometric information of $\sim 100$ bright early-type galaxies to study the galaxy light distribution out to $\sim 15-20$ effective radii. These galaxy regions are still almost unexplored in the CCD era, mainly because of the difficulties posed by the reduced detector field-of-view. The coupling of a dedicated survey telescope, the VST (Capaccioli \& Schipani 2011), with a new-generation wide-field optical imager, the OmegaCAM (Kuijken 2011), offers a great opportunity to investigate this issue. Similar studies for the northern hemisphere are being carried out for the "Next generation Virgo cluster survey" (NGVS; Ferrarese et al. 2012), and the "Mass assembly of early-type galaxies with their fine structures" survey (MATLAS; Duc 2014).

The specific aim of the VEGAS-SSS series is to study and characterize the properties of the SSSs out to very large galactic radii, taking advantage of VEGAS imaging data. Small stellar systems, especially the GC systems, have been studied for decades, and progress has been limited not so much by telescope collecting area, but by the field of view and the image quality (both to reduce contamination and to reduce the exposure times). Thus, the use of $8 \mathrm{~m}$ and even $4 \mathrm{~m}$ telescopes is not compelling, at least for the photometry. In this paper we show the original achievements possible with wide-field imaging from a $2.6 \mathrm{~m}$ telescope.
So far, except for the already mentioned ongoing studies from the NGVS and MATLAS surveys, the SSSs field population of only a few galaxies has been analyzed out to large galactocentric radii (Dirsch et al. 2003; Forbes et al. 2011; Usher et al. 2012; Blom et al. 2012), although typical studies did not go much beyond $30^{\prime} \times 30^{\prime}$, which makes a robust estimate of the total background contamination difficult. Taking advantage of the large field of view of the VST, we here:

- analyze the photometry in $g$ and $i$ bands for candidate GCs, UCDs, ECs, dSphs, etc. Furthermore, at completion, VEGAS will also include $r$ data for most of the targets, and $u$ for selected galaxies;

- study the properties of various SSS populations as a function of galactocentric distance to limits not reached to date;

- when possible, characterize the spatial extent of sources, with the specific purpose of increasing the efficiency in distinguishing between the various classes of SSSs;

- provide catalogs of SSS candidates, which are essential for preparing spectroscopic follow-up campaigns based on samples suffering for low or, at least, controlled fore- or background contamination. To this aim, VEGAS-SSS data covering the $u$ bands, possibly complemented with near-IR photometry, would be particularly efficient (Muñoz et al. 2014).

Here, we present the analysis of the $g$ and $i$ band of the field centered on NGC 3115 , with the aim of describing the data reduction, the analysis tools, and performances of the telescope, and to anticipate the future exploitation of the survey. In particular, we mostly focus on the properties of the GC system in the galaxy. Throughout the paper we verify the reliability of the methodologies used by taking advantage of the large amount of literature data available for NGC 3115 (including results from HST observations and 8-10 m class telescopes) and present original results on SSSs topics made possible by the use of the largeformat CCD mosaic. Indeed, NGC 3115, an isolated lenticular galaxy, is particularly interesting for testing the procedures used. Because of its proximity, the galaxy and its satellites were targeted by many photometric and spectroscopic studies (Hanes \& Harris 1986; Capaccioli et al. 1987; Kundu \& Whitmore 1998; Puzia et al. 2000, 2002; Norris et al. 2006; Arnold et al. 2011; Usher et al. 2012). Moreover, we recall that the GC system of the galaxy is the first system beyond the Local Group with a confirmed bimodal metallicity distribution, as shown by Brodie et al. (2012) from calcium triplet analysis and by Cantiello et al. (2014) using optical to near-IR photometry (see also Blakeslee et al. 2010; Yoon et al. 2011).

The paper is organized as follows: the next section briefly describes the observations and data reduction procedures. We introduce in Sect. 3 the data analysis and the full catalog, which provides the details of the photometric and morphological study of SSS candidates. Taking advantage of the large field-of-view of the images, we study in Sect. 4 the properties of the GC population versus galactocentric radius using a statistical background decontamination method. Section 5 is dedicated to the delicate matter of deriving SSS sizes. The final section provides a summary of our main conclusions and describes the perspectives for the forthcoming VEGAS-SSS studies.

\section{Observations and data reduction}

The VLT Survey Telescope VST is a wide-field optical-imaging telescope with a 2.6-m aperture, operating from the $u$ to the $z$ 


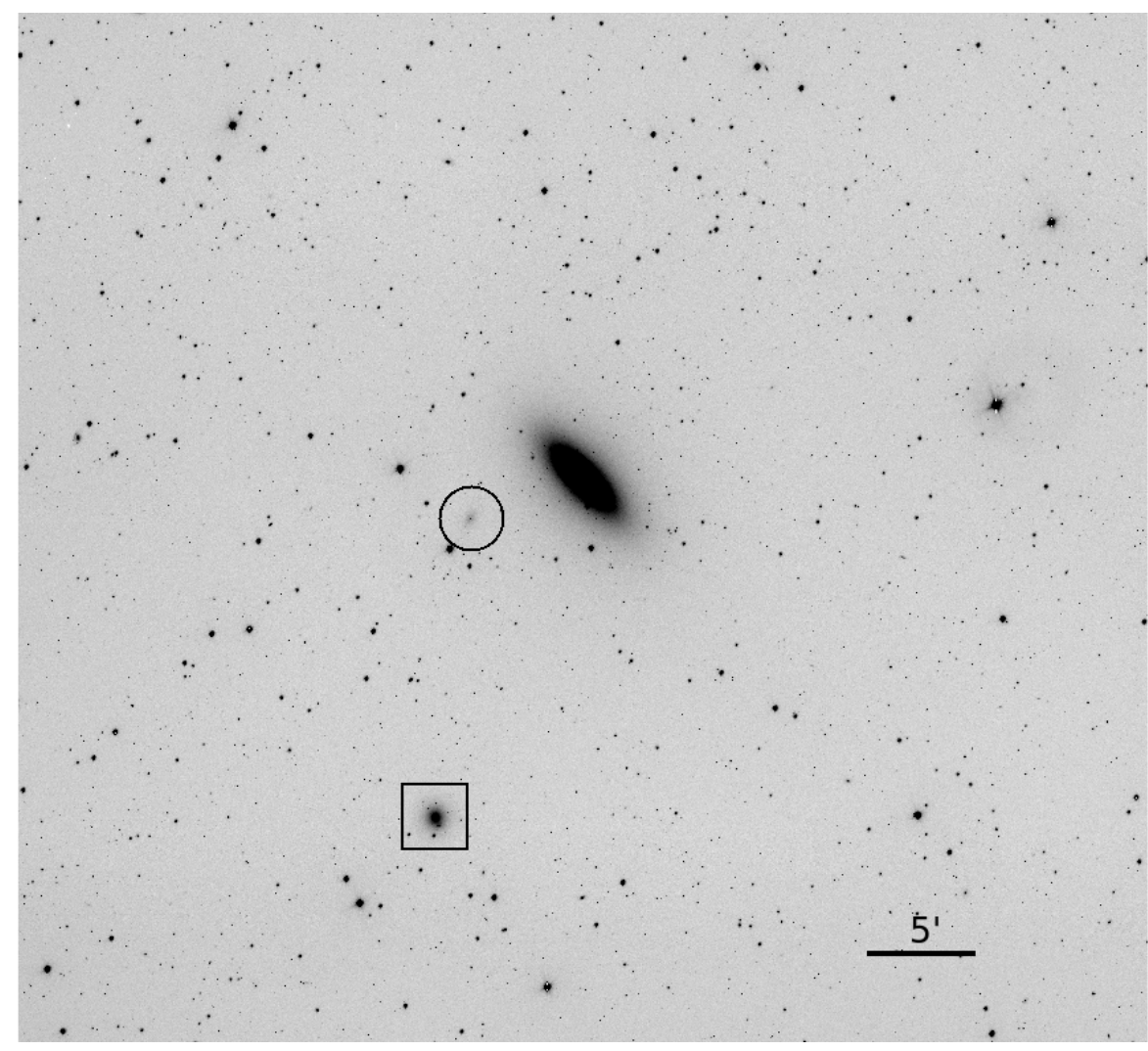

Fig. 1. VST/OmegaCAM $g$-band image of NGC 3115. North is up, east is left. The image size is $52.5^{\prime} \times 52.5^{\prime}$. The black square and circle mark the position of NGC $3115 \mathrm{~B}$ and KK 084. with a corrected field of view of 1 degree by 1 degree. Its single focal-plane instrument, OmegaCAM, is a large-format $(16 \mathrm{k} \times$ $16 \mathrm{k}$ pixels) CCD camera with a pixel scale of 0.21 pixel $^{-1}$.

The data reduction, including dither combination, vignetting, and exposure correction, astrometric solution, and photometric calibration was performed with the VST-Tube pipeline (Grado et al. 2012). Details about the overall data quality can be found in Capaccioli et al. (in prep.). In particular, the full width at half maximum (FWHM) of the point spread function (PSF) varies for $<0$ '. 05 across the field of view, and the internal astrometric accuracy is $\sim 0$.'035 (the rms with respect to the USNO-B1 catalog is $\sim 0$ '. 2).

To improve the analysis of the spatial extent of the sources in the frame, we restricted our analysis to imaging data with an average PSF $F W H M \leq 0{ }^{\prime} 8$. With this choice the exposure time is reduced by $\sim 30 \%$ in $g$ and $\sim 50 \%$ in $i$ with respect to the total integration time available.

Basic properties for the target and optical observations are listed in Table 2. The $g$-band image of NGC 3115 is shown in Fig. 1.

To study SSSs, we need to minimize the contamination due to the light from NGC 3115. To model and subtract the galaxy, we used the ISOPHOTE/ELLIPSE task in IRAF/STSDAS (Jedrzejewski 1987) ${ }^{1}$. The modeling failed to match the central thick-disk region, which implied poor detection of the sources

\footnotetext{
1 IRAF is distributed by the National Optical Astronomy Observatory, which is operated by the Association of Universities for Research in Astronomy (AURA) under cooperative agreement with the National Science Foundation.
}

Table 2. Main properties of NGC 3115.

\begin{tabular}{|c|c|}
\hline \multicolumn{2}{|c|}{ Galaxy parameters } \\
\hline $\mathrm{RA}(\mathrm{J} 2000)^{1}$ & $10 \mathrm{~h} 05 \mathrm{~m} 14.0 \mathrm{~s}$ \\
\hline $\operatorname{Dec}(J 2000)^{1}$ & $-07 \mathrm{~d} 43 \mathrm{~m} 07 \mathrm{~s}$ \\
\hline Galaxy type ${ }^{2}$ & SO \\
\hline Distance adopted (Mpc) & 9.4 \\
\hline Absolute $B$-band magnitude ${ }^{2}$ & -19.9 \\
\hline$c z^{1}\left(\mathrm{~km} \mathrm{~s}^{-1}\right.$, heliocentric $)$ & $663 \pm 4$ \\
\hline Mean $E(B-V)^{3}$ & 0.042 \\
\hline Effective radius $R_{\text {eff }}$ & $57^{\prime \prime}$ \\
\hline \multicolumn{2}{|c|}{ Observations } \\
\hline Filter (median $F W H M$ ) & Exposure time (s) \\
\hline$g\left(\sim 0.75^{\prime \prime}\right)$ & 2695 \\
\hline$i\left(\sim 0.72^{\prime \prime}\right)$ & 1250 \\
\hline
\end{tabular}

Notes. (1) Data retrieved from NED, nedwww.ipac.caltech.edu; (2) Hyperleda, leda.univ-lyon1.fr; ${ }^{(3)}$ Schlegel et al. (1998) with the recalibration of Schlafly \& Finkbeiner (2011).

within the inner $\sim 2^{\prime}$ area $^{2}$. However, the central regions of the galaxy were accurately inspected using a $\sim 10^{\prime} \times 7^{\prime}$ mosaic

2 A test with GALFIT (Peng et al. 2002), another program designed for modeling two-dimensional brightness profiles, also failed to model the central galaxy regions. We also obtained a galaxy-subtracted frame as described in Jordán et al. (2007) and Cantiello et al. (2014, which modeled NGC 3115 from near-IR data). This method uses the SExtractor spline background derived from the image logarithm, and provided very flat residuals. However, the latter procedure affects the shape of slightly extended objects, which makes it unsuitable for the purposes of the present study. 


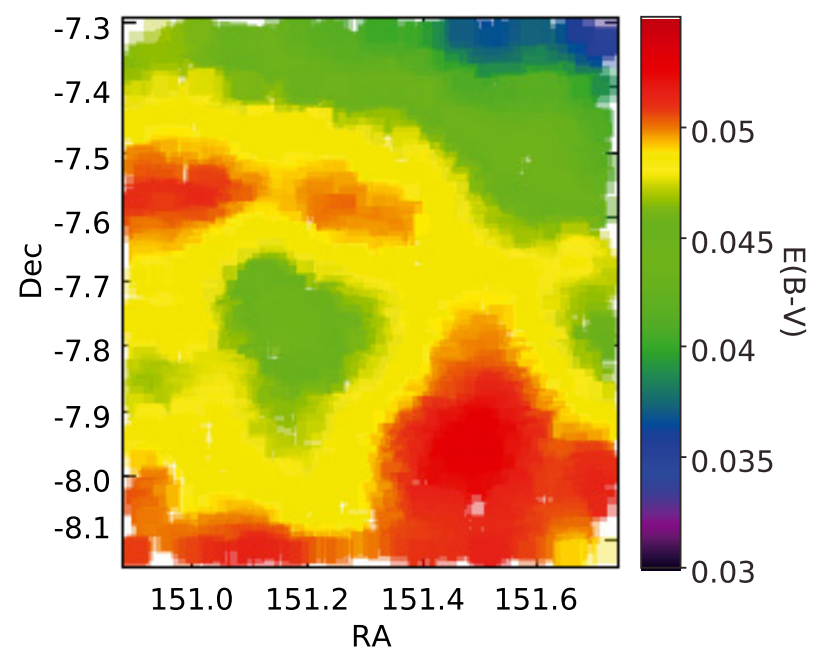

Fig. 2. Extinction map of the area. The color bar shows the extinctionto-color mapping.

obtained with the ACS camera onboard of the Hubble Space Telescope (HST; Jennings et al. 2014). The ACS study relies on data with similar wavelength coverage and $g$-band depth as the data used here. Given the higher resolution of HST data, we did not attempt to recover the sources in the poorly modeled central $\sim 3.5^{\prime} \times 1.5^{\prime}$ regions along the galaxy major axis.

\section{Photometry and size estimates}

To produce a complete catalog of all sources in the VST field of view, we independently ran SExtractor (Bertin \& Arnouts 1996) on the galaxy-model-subtracted frame for each filter.

We obtained aperture magnitudes within a diameter aperture of six pixels ( $\sim 1$ '.26 at OmegaCAM resolution), and applied aperture correction to infinite radius. The aperture correction, derived from the analysis of the curve of growth of bright isolated point-like sources (see Cantiello et al. 2005, 2011, for more details), is $0.52 \pm 0.01$ and $0.46 \pm 0.01 \mathrm{mag}$ in $g$ and $i$. For extended sources, that is, for sources that are spatially more extended than the instrumental FWHM of the PSF (see below), we used the SExtractor Kron-like elliptical aperture magnitude. Finally, the photometric catalogs in the two bands were matched adopting 0 .'5 matching radius. The final photometric catalog contained $\sim 47000$ sources.

As a result of the large areal coverage, there is a nonnegligible variation of Galactic extinction from one side to the other of the field ( $\left.\Delta A_{g} \sim 0.07 \mathrm{mag}\right)$. We obtained the local extinctions from the dust maps by Schlegel et al. (1998) and used the reddening factors from Schlafly \& Finkbeiner (2011). The final extinction map is shown in Fig. 2. All colors and magnitudes are corrected for extinction unless otherwise stated. Other details on the photometric properties of the images analyzed (completeness and limiting magnitudes) are given in Appendix A.

The color-magnitude diagram of the full sample of $g$ and $i$ matched sources is shown in Fig. 3 (panel a).

It is very important to emphasize that the selection of SSSs based on one single color, the $(g-i)$, is inherently uncertain and results in a catalog with large fractions of contaminating sources (foreground stars and background galaxies, Durrell et al. 2014). The selection with an additional optical color would certainly reduce the fraction of contaminants, especially if $u$-band photometry is available. However, a contaminant-free catalog based on optical photometry is basically unattainable. It is useful to highlight, however, that the coupling of optical data with only one near-IR band is very effective in reducing the fraction of contamination to the GC and UCD catalogs to lower than $~ 5 \%$ (Muñoz et al. 2014).

To partly overcome the problem of selecting SSSs by relying on one optical color alone, one can use statistical decontamination techniques (see Sect. 4), and/or add another selection criterion: the physical extent of the source (Table 1). The methodology we adopted to derive object sizes is described below, while the effectiveness and the practical problems in using object sizes as a selection parameter are discussed in Sect. 5 .

\subsection{Size and shape measurements as compactness criterion}

As shown in Table 1, when the half-light radius $R_{\mathrm{h}}$ of SSSs can be estimated, then the object shape can be used together with photometric properties to classify the system. However, size measurements can be very challenging, especially with groundbased imaging data. Furthermore, only angular sizes can be measured in general, which require the previous knowledge of the object distance to be transformed in linear scale. In spite of this, angular sizes and shapes have been estimated for a large sample of SSSs in different environments and with various groundand space-based telescopes (e.g. Larsen 1999; Larsen \& Brodie 2003; Jordán 2004; Cantiello et al. 2007; Caso et al. 2013; Puzia et al. 2014). Below we describe how object sizes were estimated for objects in the VEGAS-SSS fields.

Given the difficulty posed by the task, specific tools were designed and implemented to analyze the light profiles of sources with intrinsic sizes similar to or slightly smaller than the instrumental PSF, to estimate the intrinsic size of a source exceeding some instrumental-dependent size limit. For VEGAS-SSS we choose to adopt Ishape ${ }^{3}$ to obtain structural parameters (in particular $R_{\mathrm{h}}$ and the axis ratio $b / a$ ) of SSSs. Ishape is optimized for modeling the light distribution for marginally resolved sources down to $1 / 10$ of the FWHM of the PSF (Larsen 1999; Larsen \& Richtler 2000). In this context, NGC 3115 is one of the most attractive targets in the survey, being also one of the nearest. At the adopted distance of 9.4 Mpc (Tonry et al. 2001, using the updated calibration zeropoint from Cantiello et al. 2013), and given the FWHM of the images (Table 2), Ishape can be used to determine the physical extent of objects with $R_{\mathrm{h}} \geq 3.5 \mathrm{pc}$. For the reasons explained in Sect. 5, we also took into account objects down to $R_{\mathrm{h}} \sim 2$ pc.

Measuring source size below the FWHM is particularly demanding in terms of the signal-to-noise ratio $(\mathrm{S} / \mathrm{N})$. We checked that the currently available $i$-band data did not provide an adequate $\mathrm{S} / \mathrm{N}$, hence we used the $R_{\mathrm{h}}$ estimates derived from the $g$ band throughout.

Ishape reaches a convergence for $\sim 30000$ of the input $\sim 47000$ sources in the photometric catalog. The size-color and size-magnitude plots for the sample of sources for which we have structural parameters are shown in Fig. 3 (panels b and c).

More details on Ishape runs are given in Appendix B.

\subsection{Final catalog}

The final catalog resulting from the color, size, and shape criteria is given in Table 3. The catalog contains the full list of $\sim 47000$ sources matched in the $g$ and $i$ catalogs. For each source, the following parameters are reported: Col. 1 VEGASSSS ID; Cols. 2 and 3 right ascension and declination (J2000);

3 The software can be downloaded at http://baolab.astroduo. org/. We used release $0.94 .1 \mathrm{~d}$. 

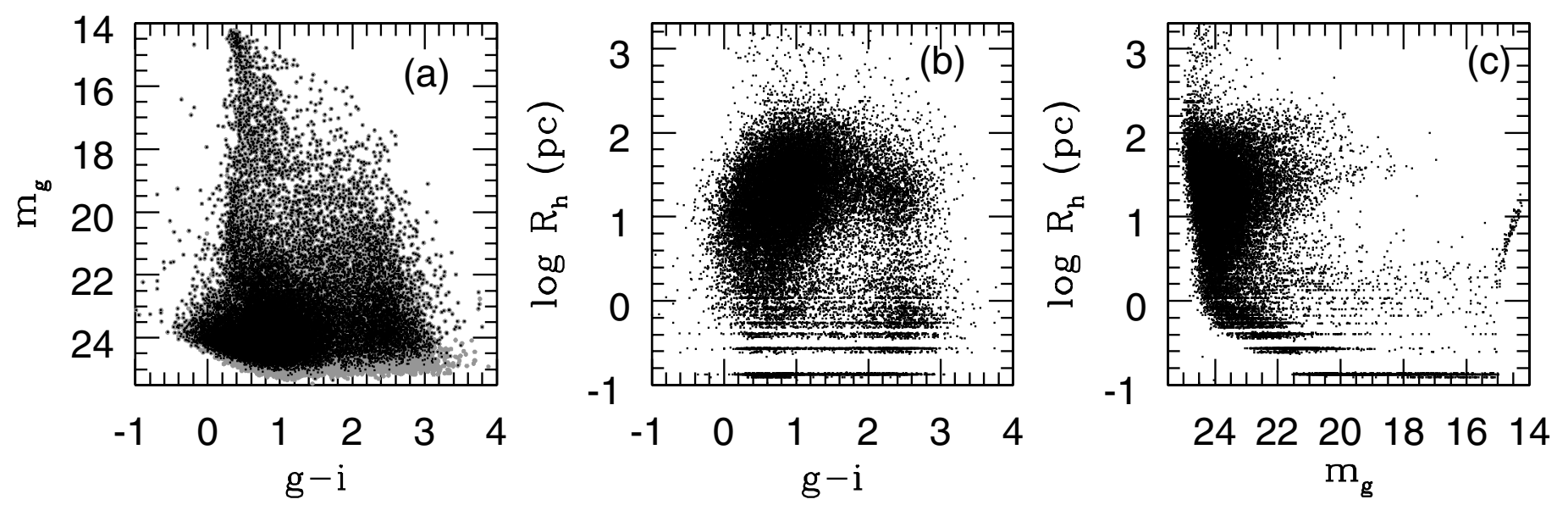

Fig. 3. Photometry and sizes of SSS candidates in NGC 3115. Panel a) color-magnitude diagram for the full sample of $g$ and $i$ matched sources (gray dots) and for the sources with size estimates (black points). Panel b) apparent size-versus-color diagram. Panel c) apparent size-versusmagnitude diagram.

Table 3. Photometry and size estimates for the matched gi catalogs.

\begin{tabular}{|c|c|c|c|c|c|c|c|c|c|c|c|c|c|c|}
\hline \multicolumn{4}{|c|}{ Position } & \multicolumn{5}{|c|}{ Photometry } & \multicolumn{6}{|c|}{ Ishape results } \\
\hline ID & $\begin{array}{c}\text { RA } \\
(\mathrm{J} 2000)\end{array}$ & $\begin{array}{c}\text { Dec } \\
(\mathrm{J} 2000)\end{array}$ & $\begin{array}{l}R_{\text {gal }} \\
\left({ }^{\prime}\right)\end{array}$ & $\begin{array}{c}m_{g} \\
(\mathrm{mag})\end{array}$ & $C S_{g}$ & $\begin{array}{c}m_{i} \\
(\mathrm{mag})\end{array}$ & $C S_{i}$ & $(g-i)$ & $E(B-V)$ & $S / N$ & $\begin{array}{c}F W H M \\
\left({ }^{\prime \prime}\right)\end{array}$ & $\begin{array}{c}R_{\mathrm{h}} \\
(\mathrm{pc})\end{array}$ & $b / a$ & Note $^{a}$ \\
\hline (1) & (2) & (3) & (4) & (5) & (6) & $(7)$ & (8) & (9) & (10) & (11) & $(12)$ & (13) & (14) & (15) \\
\hline 4 & 151.040149 & -8.155928 & 30.7 & $25.35 \pm 0.20$ & 0.45 & $24.10 \pm 0.23$ & 0.56 & 1.250 & 0.053 & $\ldots$ & $\ldots$ & $\ldots$ & $\ldots$ & 0 \\
\hline 6 & 151.484945 & -8.155959 & 28.3 & $24.51 \pm 0.09$ & 0.70 & $23.91 \pm 0.19$ & 0.72 & 0.602 & 0.052 & $\ldots$ & $\ldots$ & $\ldots$ & $\ldots$ & 0 \\
\hline 92 & 151.348259 & -8.155089 & 26.3 & $23.97 \pm 0.10$ & 0.02 & $21.86 \pm 0.08$ & 0.06 & 2.109 & 0.051 & 18.6 & $0.95_{-0.95}^{0.33}$ & 12.5 & 0.85 & 0 \\
\hline 93 & 151.091210 & -8.154886 & 29.2 & $24.26 \pm 0.12$ & 0.39 & $23.05 \pm 0.14$ & 0.38 & 1.210 & 0.053 & 11.4 & $1.14_{-1.14}^{0.11}$ & 15.0 & 0.86 & 0 \\
\hline 293 & 151.571415 & -8.153376 & 30.4 & $21.78 \pm 0.02$ & 0.03 & $21.27 \pm 0.04$ & 0.03 & 0.502 & 0.051 & 106.7 & $2.49_{-0.09}^{0.03}$ & 34.8 & 0.97 & 1 \\
\hline 993 & 151.117979 & -8.143415 & 27.9 & $21.86 \pm 0.02$ & 0.03 & $21.19 \pm 0.04$ & 0.04 & 0.675 & 0.053 & 94.8 & $1.74_{-0.10}^{0.02}$ & 22.0 & 0.78 & 1 \\
\hline 1396 & 150.878854 & -8.138160 & 35.8 & $22.37 \pm 0.02$ & 0.86 & $21.67 \pm 0.03$ & 0.26 & 0.700 & 0.052 & 64.3 & $0.53_{-0.01}^{0.08}$ & 6.7 & 0.77 & 2 \\
\hline
\end{tabular}

Notes. The full table is available at the CDS. ${ }^{(a)} 0$ : source common to both $g$ and $i$ catalogs without Ishape data, or rejected from the reference and best samples; 1) source in the reference sample; 2) source in the best sample.

Col. 4 galactocentric distance; Col. $5 \mathrm{~g}$ magnitude and error; Col. 6 SExtractor CLASS_STAR parameter in the $g$ band CS $_{g}$; Col. $7 i$ magnitude and error; Col. 8 SExtractor CLASS_STAR parameter in the $i$ band $\mathrm{CS}_{i}$; Col. $9(g-i)$ color; Col. 10 local reddening; Col. $11 \mathrm{~S} / \mathrm{N}$ from Ishape; Col. 12 FWHM of the source; Col. 13 effective radius; Col. 14 object minor to major axis ratio $(b / a)$; Col. 15 notes. The absolute value of $R_{\mathrm{h}}$ in pc depends on the distance adopted, thus it is incorrect for all unknown contaminating fore- and background sources. In Sect. 5 we discuss the percentage of contamination expected on the basis of a comparison with data from the literature.

\section{GC population properties as a function of galactocentric distance: statistical decontamination of the sample}

In this section, we analyze the color and magnitude distribution of SSSs in the field of NGC 3115. Because they dominate the SSS population in the galaxy core, we focus in particular on GCs, making use of statistical decontamination of background sources. To have a better statistics for the background subtraction, we used the entire VEGAS-SSS catalog of $g$ and $i$ matched sources ( 47000 objects), and selected good GC candidate sources a) in the color range for $0.4 \leq(g-i) \leq 1.25 \mathrm{mag}$ (e.g.,
Faifer et al. 2011; Kartha et al. 2014; Vanderbeke et al. 2014); b) maximum photometric error $\Delta(g-i)=0.15$ mag for color analysis ( $\Delta m_{g}=0.5 \mathrm{mag}$ for magnitude analysis); c) SExtractor star-galaxy $\langle\mathrm{CS}\rangle \geq 0.2$, to avoid contamination from sources that are trivial background galaxies; d) and $m_{g} \geq 18$ mag, that is, sources $\sim 4 \sigma_{\text {TOM }}$ brighter than $m_{g}^{\text {TOM }}$ were not taken into account (see below). Thus, for the analysis presented in this section we did not apply any restriction on $R_{\mathrm{h}}$.

\subsection{Background determination}

Our approach relies on the assumption that all sources beyond some limiting galactocentric radius, $R_{\mathrm{bg}}$, are foreground or background contaminants, that are uniformly spatially distributed across the field. In particular, we adopted $R_{\mathrm{bg}}=23^{\prime}$ (see also Sect. 5), which corresponds to $\sim 65 \mathrm{kpc}$ at the distance of the galaxy. Taking as reference the GC systems in the Milky Way and M31, we estimate that a fraction of $\sim 2-3 \%$ GCs brighter than $m_{g} \sim 25$ mag (the approximate $90 \%$ limiting magnitude in $g$, see Fig. 3 and Appendix A) might still be in the background sample because of their large galactocentric distances $R_{\text {gal }} \gtrsim 65 \mathrm{kpc}$. The Galaxy has seven GCs, over 157 , at $R_{\text {gal }} \gtrsim 65 \mathrm{kpc}$ (Harris 1996, 2010 release), only three of them are brighter than the detection limit of our photometric catalog. This 


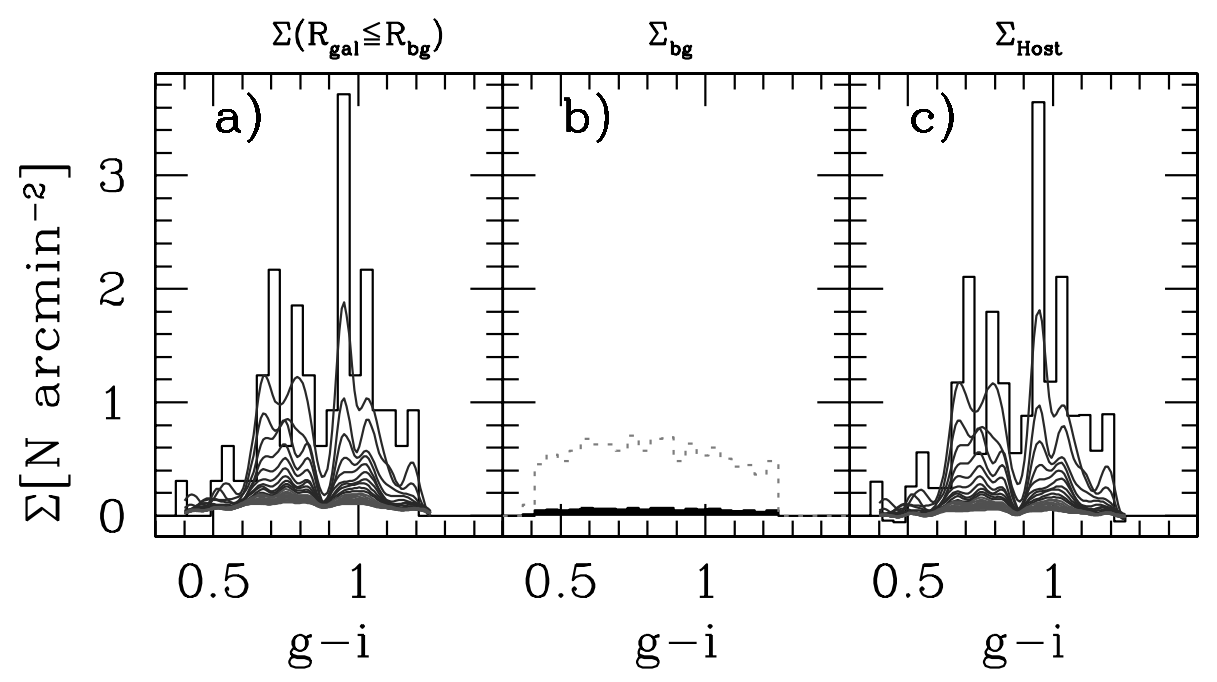

Fig. 4. Surface density histograms versus color Panel a) Surface density for sources within $R_{\text {gal }} \leq R_{\text {bg }}$. Darker color refers to areas with smaller galactocentric radii. Panel b) same as left, but for background sources at $R_{\text {gal }}>$ $R_{\mathrm{bg}}$. For the sake of clarity, the dotted histogram shows the density histogram times a factor 10 . Panel c) residual surface density, $\Sigma\left(R_{\mathrm{gal}} \leq R_{\mathrm{bg}}\right)-\Sigma_{\mathrm{bg}}$. implies that, if placed at the distance of NGC 3115, and for random viewing angles, $\$ 2 \%$ of the MW GCs would be included in the background sample. The GCs catalog of M 31 by Galleti et al. (2004; RBCv5), selected using optical to near-IR color cuts (Muñoz et al. 2014), contains 447 GC candidates, none of which at galactocentric distance larger than $35 \mathrm{kpc}$. On the other hand, Huxor et al. (2014) discovered 59 new GCs at large galactocentric distances in Andromeda using CFHT/MegaCam data of the PAndAS survey: 19 of them would be brighter than our magnitude cut and are located at a projected distance larger than $65 \mathrm{kpc}$ from the galaxy center. This corresponds to a fraction of $\sim 3 \%$ of the total, assuming a total population of at least $700 \mathrm{GCs}$ (638 from RBCv5, 59 from PAndAS).

Adopting $R_{\mathrm{bg}}=23^{\prime}$ means that $\sim 40 \%$ of the image is used to analyze contamination. The possible future addition of more bands will allow increasing the inner radius for the selection of GCs (more in general, of SSS satellites), which will allow using a smaller fraction of the detector to characterize the contamination.

Under this assumption, the difference between the surface density at galactocentric distance $R_{\mathrm{gal}} \leq R_{\mathrm{bg}}$ and $R_{\mathrm{gal}}>R_{\mathrm{bg}}$ gives the residual density of sources in NGC 3115 , mainly GCs.

We proceeded as follows: we first estimated the surface density of background objects per square arcminute at a given color (or magnitude), $\Sigma_{\text {bg }}$, by adopting the selection criteria (a)-(d) given above, plus the galactocentric distance. Then, the total surface density of objects within elliptical concentric regions, $\Sigma\left(R_{\text {gal }} \leq R_{\text {bg }}\right)$, was estimated using the same criteria on color (or magnitude), adopting different inner radii, starting from $R_{\text {gal }}=$ $2^{\prime}$ out to $R_{\mathrm{gal}}=R_{\mathrm{bg}}$, with $1^{\prime}$ steps. The geometry of the ellipses, with constant ellipticity $\epsilon=0.5$ and position angle $\mathrm{PA}=45^{\circ}$, was assumed following the results of Capaccioli et al. (in prep.) (see also Arnold et al. 2011; Jennings et al. 2014). In the following, $R_{\text {gal }}$ is the semimajor axis, if not stated otherwise.

The overdensity of sources at given colors (or magnitude) associated with NGC 3115 is finally estimated as the difference $\Sigma_{\mathrm{Host}} \equiv \Sigma\left(R_{\mathrm{gal}} \leq R_{\mathrm{bg}}\right)-\Sigma_{\mathrm{bg}}$.

\subsection{Color distribution}

The panels in Fig. 4 show the density histograms $\Sigma\left(R_{\mathrm{gal}} \leq R_{\mathrm{bg}}\right)$, $\Sigma_{\mathrm{bg}}$, and $\Sigma_{\text {Host }}$ versus color (from left to right, respectively). In each panel, darker color refers to regions with smaller inner radii. The histograms after the first innermost radius were
Table 4. Results of GMM at various galactocentric radii.

\begin{tabular}{cccc}
\hline \hline$\left.R_{\text {gal }}{ }^{\prime}\right)$ & $(g-i)_{0}^{\text {blue }}$ & $(g-i)_{0}^{\text {red }}$ & $N_{\mathrm{GC}}^{\text {sel }}$ \\
\hline 2.0 & $0.79 \pm 0.18(0.64)$ & $1.01 \pm 0.07(0.30)$ & 68 \\
3.0 & $0.78 \pm 0.15(0.69)$ & $1.00 \pm 0.06(0.26)$ & 150 \\
4.0 & $0.75 \pm 0.14(0.64)$ & $1.02 \pm 0.07(0.30)$ & 204 \\
5.0 & $0.76 \pm 0.14(0.74)$ & $1.01 \pm 0.05(0.19)$ & 268 \\
6.0 & $0.76 \pm 0.14(0.70)$ & $1.01 \pm 0.05(0.23)$ & 315 \\
7.0 & $0.76 \pm 0.14(0.70)$ & $1.02 \pm 0.06(0.25)$ & 364 \\
8.0 & $0.76 \pm 0.13(0.65)$ & $1.02 \pm 0.06(0.29)$ & 400 \\
9.0 & $0.75 \pm 0.13(0.65)$ & $1.01 \pm 0.06(0.29)$ & 454 \\
10.0 & $0.75 \pm 0.13(0.65)$ & $1.00 \pm 0.05(0.23)$ & 518 \\
11.0 & $0.76 \pm 0.13(0.66)$ & $1.00 \pm 0.06(0.24)$ & 562 \\
12.0 & $0.75 \pm 0.13(0.67)$ & $1.00 \pm 0.06(0.27)$ & 612 \\
13.0 & $0.75 \pm 0.13(0.69)$ & $1.00 \pm 0.05(0.22)$ & 661 \\
14.0 & $0.75 \pm 0.14(0.69)$ & $1.00 \pm 0.05(0.21)$ & 718 \\
15.0 & $0.75 \pm 0.13(0.70)$ & $1.00 \pm 0.05(0.23)$ & 768 \\
16.0 & $0.74 \pm 0.14(0.69)$ & $1.01 \pm 0.08(0.29)$ & 833 \\
17.0 & $0.75 \pm 0.14(0.70)$ & $1.02 \pm 0.07(0.28)$ & 890 \\
18.0 & $0.75 \pm 0.13(0.69)$ & $1.00 \pm 0.05(0.23)$ & 963 \\
19.0 & $0.75 \pm 0.13(0.69)$ & $1.00 \pm 0.05(0.22)$ & 1024 \\
20.0 & $0.75 \pm 0.13(0.69)$ & $1.01 \pm 0.06(0.24)$ & 1086 \\
21.0 & $0.75 \pm 0.14(0.70)$ & $1.00 \pm 0.05(0.22)$ & 1159 \\
22.0 & $0.73 \pm 0.14(0.66)$ & $1.01 \pm 0.08(0.31)$ & 1247 \\
\hline
\end{tabular}

smoothed for the sake of clarity. In the first panel of the figure, the density distribution shows a dip at $(g-i) \sim 0.9$ mag and two well-defined peaks at $(g-i) \sim 0.75$ and 1.00 mag whose prominence decreases, but does not reach zero as larger radii are considered.

For background sources (Fig. $4, \Sigma_{\text {bg }}$ middle panel) the density distribution does not show relevant features and appears to be nearly flat in the color interval shown. As expected, the color distribution of the difference diagram (Fig. $4, \Sigma_{\text {Host }}$ right panel) shows two distinct color peaks at all radii.

To investigate the properties of the color distributions in panel (c) at each given radius, we used the Gaussian mixture modelling code (GMM; Muratov \& Gnedin 2010) ${ }^{4}$. More

4 GMM uses the likelihood-ratio test to compare the goodness of fit for double-Gaussians versus a single-Gaussian. For the best-fit double model, it estimates the means and widths of the two components, their separation DD in terms of combined widths, and the kurtosis of the overall distribution. It also provides uncertainties based on bootstrap resampling. In addition, the GMM analysis provides the positions, relative widths, and fraction of objects associated with each peak. 


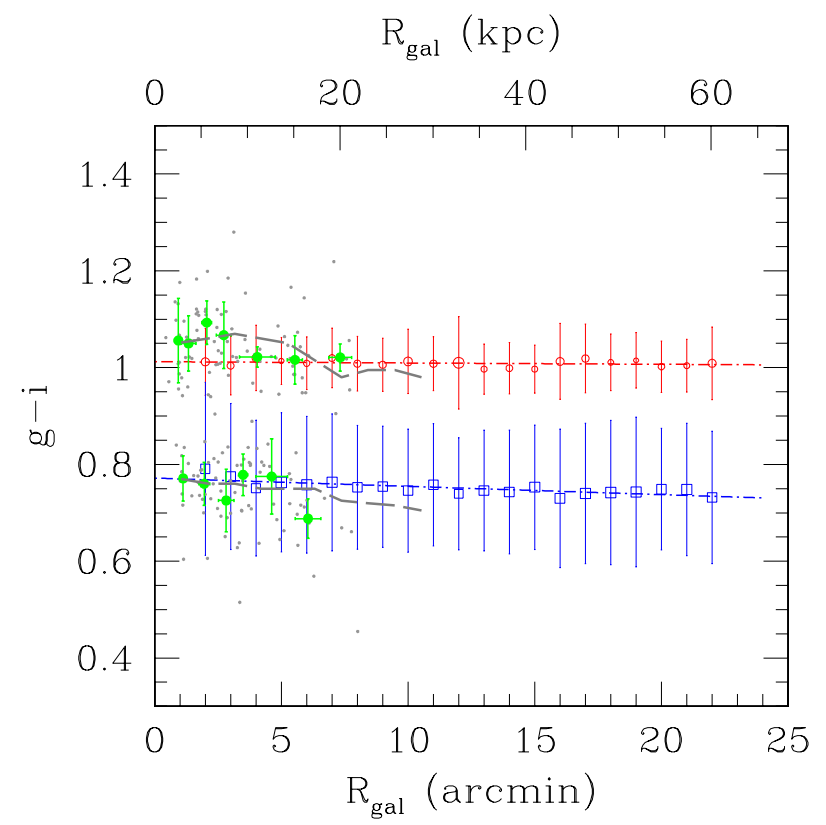

Fig. 5. Position and width of the blue and red GCs (blue squares and red empty circles) at different $R_{\text {gal }}$ as obtained from GMM. Symbol size is proportional to the fraction of objects associated with each peak. A fit to the data is shown with dot-dashed lines for the two subpopulations. Gray dots show spectroscopic confirmed GCs from Arnold et al. (2011). Full green points mark the running mean (median from equal number of data) of gray points. Gray long-dashed lines mark the rolling fits of the blue and red GC peaks as derived by Arnold et al. (2011) obtained from a combination of spectroscopically and photometrically selected GCs.

specifically, we randomly populated the difference distribution, $\Sigma_{\text {Host }}$, with a fixed number of sources $\left(N_{\text {sim }} \sim 1500\right)$, and then ran the GMM code on the repopulated sample.

The results of the GMM run are given in Table 4, where for each $R_{\text {gal }}$ we report the position of the peak and width of the blue and red distributions and the fraction of GCs associated with each populations (in parentheses). In some cases the total fraction does not equal one because of a minor very red peak. Figure 5 shows the positions of the blue and red peaks, the standard deviation of each distribution, and the fraction of objects associated with each peak (given by symbol size).

In the table we also report the number of GC candidates selected according to the (a)-(d) selection criteria given above $\left(N_{\mathrm{GC}}^{\mathrm{sel}}\right.$, column). From the average surface density of contaminants $\Sigma_{\mathrm{bg}}=0.056 \pm 0.014\left[\mathrm{~N} / \mathrm{arcmin}^{2}\right]$ and the $N_{\mathrm{GC}}^{\mathrm{sel}}$ listed, the expected number of GCs corrected for contamination at each elliptical radius can be easily derived. As an example, the area with $R_{\text {gal }} \sim 6-8^{\prime}$ roughly corresponds to the ACS area inspected by Jennings et al. (2014), and is expected to contain $\sim 310-390$ GCs, to be compared with the 360 candidates found with ACS.

We note that the position of the two peaks and their width are consistent at all radii inspected, and agree very well with the recent similar analyses on the same host galaxy (Faifer et al. 2011; Usher et al. 2012). A closer inspection of the data in Fig. 5 and Table 4 reveals important features. First, a color- $R_{\text {gal }}$ correlation is observed for the blue GC component (Pearson correlation coefficients $r_{x y} \sim-0.8$ ), with a $\sim 0.06$ mag color difference between the inner and outer region. There is no or only a very weak color-radius correlation for the red GCs $\left(r_{x y} \sim-0.25\right)$. Furthermore, the fraction of sources in the red subpopulation shows a slight but significant decrease with respect to the blue one at large radii. The width of the two sequences is relatively stable with radius, with the blue distribution being broader at all radii.

These properties support a scenario where blue GCs are associated with the galaxy halo, while red ones are more centrally concentrated and associated with the bulge stellar component in the galaxy (Kissler-Patig 1997; Côté et al. 1998; Forte et al. 2005; Liu et al. 2011).

To study the population of GCs associated with NGC 3115 excluding the GC contaminants from the neighboring fainter galaxies, we also carried out several tests by rejecting all GC candidates within 2-3' from KK 084 and NGC 3115B (see Fig. 1). The first galaxy, KK 084, is a dSph centered at $R_{\text {gal }} \sim 5.5^{\prime}$ from NGC 3115, and a non-negligible population of GC candidates with specific frequency $S_{N} \equiv N_{\mathrm{GC}} 10^{0.4\left(M_{V}+15\right)}=$ 10 (Harris \& van den Bergh 1981; Puzia \& Sharina 2008). In spite of the relatively high $S_{N}$, the net effect on the properties of the GC system in NGC 3115 is negligible. None of the sources in NGC 3115B fall in the elliptically shaped area of NGC 3115 inspected here.

In Fig. 5 we added data from Arnold et al. (2011), which are part of the SLUGGS survey (Brodie et al. 2014). The mean VEGAS-SSS color obtained with the statistical decontamination approach matches the color of the spectroscopically confirmed GCs very well.

Arnold et al. (2011) also derived the radial profiles out to $R_{\text {gal }} \sim 10^{\prime}$ by combining the spectroscopic sample with a photometric sample corrected for contamination using "an iterative Monte Carlo scheme" (gray lines in Fig. 5). The VEGASSSS and SLUGGS color profiles for blue GCs match well at all common radii.

We note that at $R_{\text {gal }} \geq 6^{\prime}$ the color profiles from Arnold et al. mostly depend on the properties of the photometric sample, thus the transition appearing in both the blue and red GC profiles at $6 \geq R_{\text {gal }} \geq 8^{\prime}$ is strongly weighted toward the photometric sample.

For the blue GCs, the difference between the mean from VST and the color reported by Arnold et al. for spectroscopically confirmed GCs at $R_{\text {gal }} \leq 6^{\prime}$ is $\Delta(g-i)^{\text {blue }}<0.01 \mathrm{mag}$. For the red GC component the difference is $\Delta(g-i)^{\text {red }} \sim 0.03$ mag.

By coupling the spectroscopic and photometric samples (gray dashed lines), Arnold et al. found that the red GCs are on average bluer at larger galactocentric distances than blue GCs. A radial trend in the red GCs from SLUGGS data mostly appears beyond $R_{\text {gal }} \sim 6^{\prime}$, where the photometric sample dominates the spectroscopic one. Furthermore, the red GC profile is nearly flat for $R_{\text {gal }} \leq 5.5^{\prime}$ and $R_{\text {gal }} \geq 7.5^{\prime}$, with a $\sim 0.07$ mag color transition in between.

Overall, the radial color profiles of GCs from the VEGASSSS and SLUGGS are consistent if one takes into account the error envelopes, the intrinsic width of the distribution at fixed $R_{\text {gal }}$, and the different analysis approaches adopted.

The good agreement appears even more striking if one takes into account that the data from Arnold et al. (2011) were obtained by coupling gri-band imaging data from Suprime-Cam at the $8.2 \mathrm{~m}$ Subaru telescope and spectroscopy from the $10 \mathrm{~m}$ Keck-II telescope with DEIMOS.

In conclusion, the comparison shown in Fig. 5 provides strong evidence in support of the efficiency of the approach adopted here to analyze the properties of the GC system out to more than $\sim 20$ galactic effective radii. It also shows that original results are obtained, even with the photometry in only two passbands, when using the wide-field imaging data from the $2.6 \mathrm{~m}$ VST telescope. 


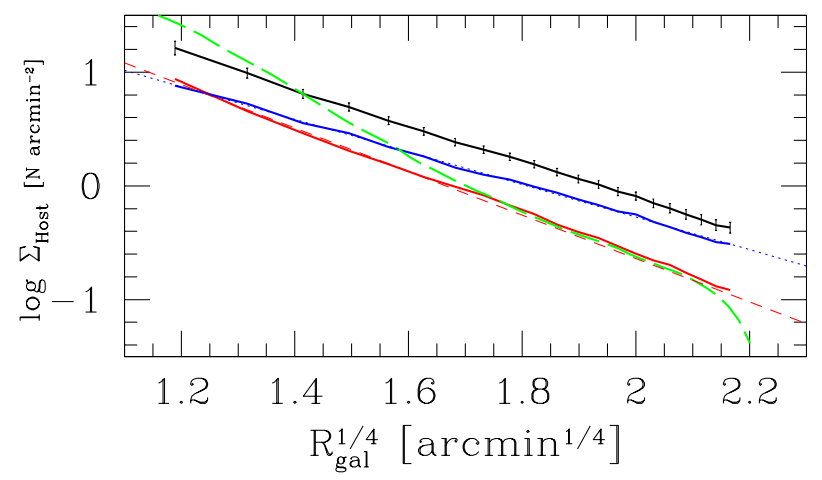

Fig. 6. Surface density profiles of blue, red, and total GC population (blue, red and black lines, respectively). The galaxy surface brightness profile in $g$ band from Capaccioli et al. (in prep.) is also reported with green long-dashed line. The linear fit to the surface density is shown with dotted lines (blue-dotted, red-dashed for the blue and red GCs). The scale of the galaxy profile is arbitrary.

\subsection{Surface density profiles}

The radial profiles of the projected surface density for GC candidates are shown in Fig. 6. The surface density at each radius is obtained as the difference between the total density of sources with $R_{\text {gal }} \leq R_{\text {bg }}$ and the background density.

Taking advantage of the results obtained with GMM on the blue and red GCs, we also analyzed the radial density profiles of the blue and red subpopulations. When the GCs are divided into subpopulations by adopting a sharp blue-to-red separation at $g-i=0.9$, the radial profile for the red GCs appears to be steeper than that for the blue GCs. Moreover, both density profiles very closely follow a $r^{1 / 4}$ de Vaucouleurs profile (dotted lines), and both are shallower than the galaxy light profile, showing a behavior similar to that of other galaxies (e.g., NGC 4636 and NGC 3923; Dirsch et al. 2005; Norris et al. 2012).

The steeper starlight gradient than seen for the GC density (blue or total GC density) suggests that the GC system of NGC 3115 extends farther than the surface brightness profile of the galaxy halo. This result is consistent with the general picture of the GC system being spatially more extended than the host galaxy (Harris 1991; Harris et al. 2000; Forbes et al. 2006; Alamo-Martínez et al. 2012; Kartha et al. 2014).

Another feature in Fig. 6 is the matching density profile for red GCs with the galaxy light profile at $R_{\text {gal }} \geq 7.5^{\prime}\left(R_{\text {gal }}^{1 / 4} \geq 1.65\right)$, while the surface density of GCs at smaller radii is slightly lower. This depletion has previously been observed in galaxies brighter than NGC 3115 (e.g., Dirsch et al. 2005; Goudfrooij et al. 2007) and is associated with the higher efficiency of GCdisruption mechanisms in the inner galaxy regions (dynamical friction, two-body relaxation, and GC tidal shocking; Vesperini 2001; Goudfrooij et al. 2007). It suggests that the galaxy has undergone a relatively quiescent evolution, without major starforming events, which would have increased the inner density of red GCs.

\subsection{Luminosity function: GCLF}

We adopted the same approach as we used for colors in Sect. 4.2 to analyze the luminosity function of sources in the field, with the specific purpose of inspecting the GC luminosity function (GCLF) to independently estimate the galaxy distance modulus (Harris 2001) and furthermore derive the position of the turnover magnitude TOM, $m_{g}^{\mathrm{TOM}}$, as a function of galactocentric distance.
Figure 7 shows the surface density distribution obtained as described in the previous section, with the difference that in this case we used the total $g$ magnitude instead of the $(g-i)$ color. The GCLFs derived were corrected for radial-dependent completeness as described in Appendix A. Panel a in the figure shows various local maxima in the density distribution, whereas the distribution of background sources in panel $\mathrm{b}$ has a power-law increase with a drop between $m_{g} \sim 24$ and $25.5 \mathrm{mag}$, due to the completeness limit given by the adopted selection criteria. The density distribution of sources in the host galaxy, shown in panel c, reveals a major peak at $m_{g} \sim 22.75 \mathrm{mag}$.

To inspect the discontinuity in the luminosity function due to the TOM, we adopted the quantitative method introduced by Lee (1993) to identify the position of the RGB tip in Galactic resolved GCs. The results of this edge-detection method (based on the Sobel filter, see Appendix A) are shown in panel d of Fig. 7. Although the uncertainties in the surface density and their propagation in the definition of the edge and second-edge functions are certainly large, the diagrams highlight an inflection point (edge) and a maximum (edge2) around $m_{g} \sim 22.75 \mathrm{mag}$, as expected at the TOM (see Fig. A. $2^{5}$ ).

In Fig. 7, we also show the Gaussian GCLF with arbitrary peak normalization, assuming a turnover magnitude $m_{g}^{\text {TOM }} \sim$ 22.75 , with $\sigma_{\mathrm{TOM}}=1.14$ derived from Jordán et al. (2009, Eq. (18)). A $\sim 0.2$ mag tolerance area around $m_{g}^{\text {TOM }}$ is also shown. We adopted the absolute value for the turnover magnitude from the ACS Virgo Cluster Survey for galaxies with $M_{B}<-18$, $M_{g}^{\mathrm{TOM}}=-7.2 \pm 0.2 \mathrm{mag}$ and estimate a distance modulus $\mu_{0}=29.95 \pm 0.3$, which agrees with the literature distance of the galaxy (Table 2).

Furthermore, thanks again to the large area inspected, we also probed the variation of $m_{g}^{\mathrm{TOM}}$ to large projected galactocentric radii. The data in Fig. 7 (panel c) reveal a TOM essentially constant over the spatial scales inspected, as also found in other galaxies (Jordán et al. 2007).

On the basis of the results shown in Figs. 7 and 4, we deduce a low contamination rate in the regions within $R_{\text {gal }} \leq 8^{\prime}$ (first six darker curves in the figures), as the luminosity and color surface density of contaminants $\Sigma_{\text {bg }}$ shown in the central panels can be one order of magnitude lower than the density in the inner regions. This implies that the contamination rate of the VEGASSSS catalog for NGC 3115 is quite low for the innermost $\sim 8^{\prime}$. As an example, the background density at $m_{g} \sim 23 \mathrm{mag}$ is $\Sigma_{\text {bg }} \sim 0.3\left[N / \operatorname{arcmin}^{2}\right]$, while the density of sources within $R_{\text {gal }} \leq 8^{\prime}$ is $\sim 3.5$ times higher and is even about seven times higher at $R_{\text {gal }} \leq 4^{\prime}$.

We also inspected how the TOM differs between red and blue GCs. This test is not often possible and feasible here thanks to the large area inspected. After dividing the blue and red GCs by adopting a sharp color separation at $(g-i)=0.9$, we carried out the analysis described above on the luminosity functions of the blue and red GCs. The results are shown in Fig. 8, where the total luminosity function $\Sigma\left(R_{\text {gal }} \leq R_{\mathrm{bg}}\right)$, the background-corrected luminosity function $\Sigma_{\text {Host }}$, and the edge and edge 2 diagrams are shown for the blue and red GCs (upper and lower panels). Even though the samples adopted are numerically smaller than before, the corrected GCLF still shows the peak around the same $m_{g}^{\mathrm{TOM}}$ of the total GC population. From estimating the position of the TOM with the edge functions (right panels in the figure), the interesting point here is that there appears to be a $\sim 0.2 \mathrm{mag}$

5 The edge functions show two other possible TOM-point candidates located at $m_{q} \sim 22.2$ and $23.2 \mathrm{mag}$ (see Appendix A). However, both magnitude values are ruled out as TOM peaks by the shape of the GCLF. 

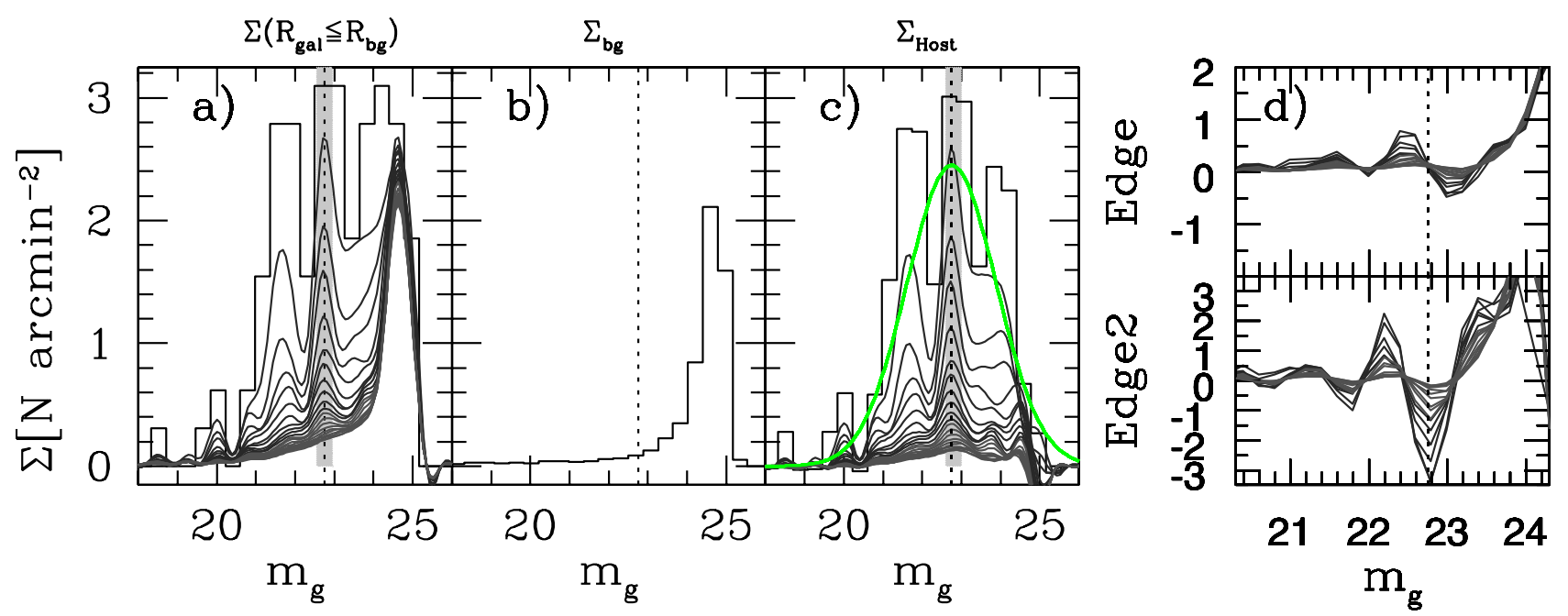

Fig. 7. Surface density histograms versus magnitude. Panels a) to c): as in Fig. 4, but magnitude is used instead of color. The vertical dotted line shows the position of the TOM. The $\sim 0.2 \mathrm{mag}$ tolerance for the peak position (gray shaded area) is also shown as well as the best-fit Gaussian to the GCLF (green line; $m_{g}^{\mathrm{TOM}}=22.75 \mathrm{mag}, \sigma_{\mathrm{TOM}}=1.14$ ). Panel d): edge and second-run edge (Edge2) functions.
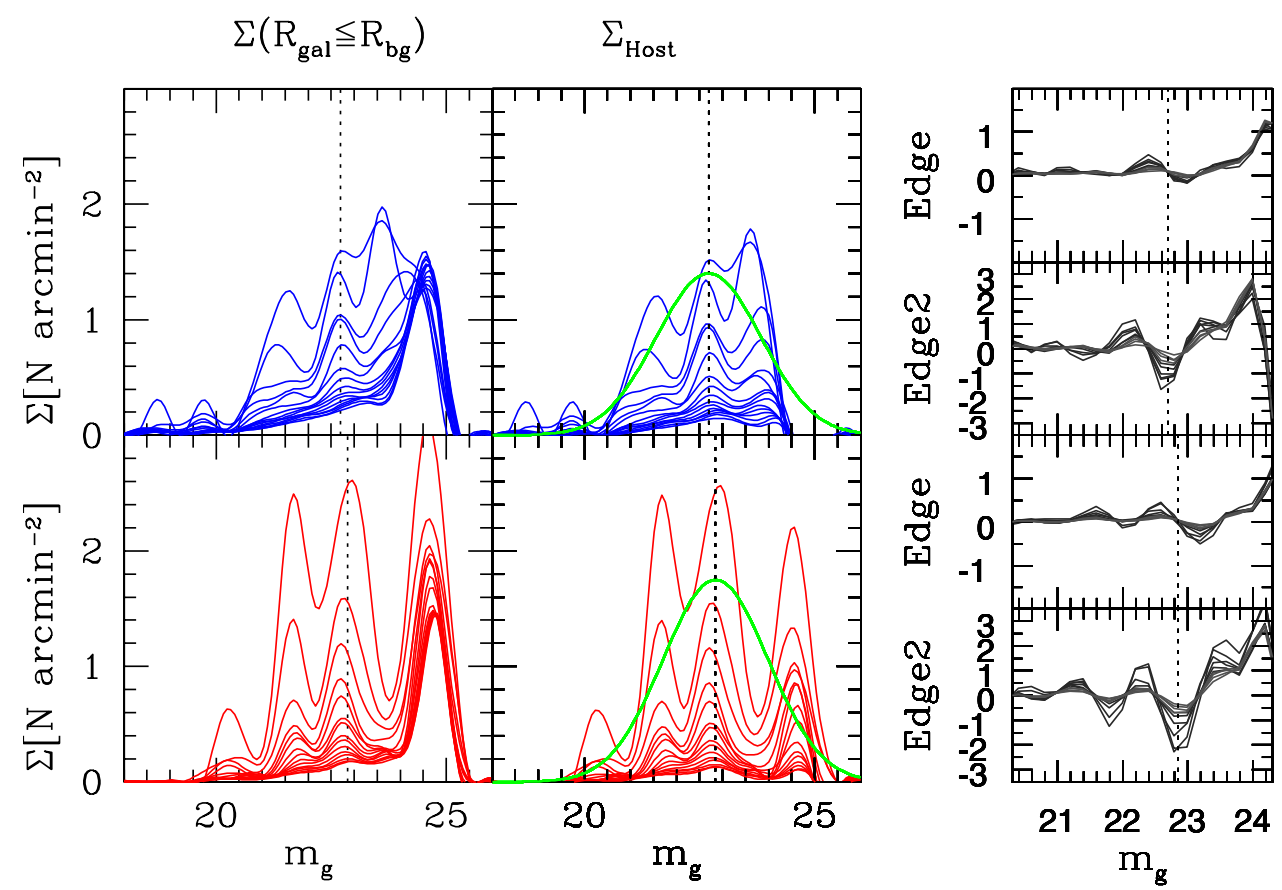

Fig. 8. GCLF for the blue and red GC components analyzed separately. The upper panels, from left to right, show the total density distribution $\Sigma\left(R_{\text {gal }} \leq R_{\mathrm{bg}}\right)$, the density after subtracting for background contamination $\Sigma_{\text {Host }}$, and the edge and edge2 functions. The vertical dashed line marks the approximate position of the TOM, as obtained from the edge functions. The green line shows a Gaussian with peak at $m_{g}^{\text {TOM }}=22.7$ mag. Lower panels: as upper ones, but for the red GC components, with $m_{q}^{\mathrm{TOM}}=22.85 \mathrm{mag}$.

offset between the TOM of red and blue GCs, with the red system being fainter. From the point of view of stellar population models, if the GCs mass function is universal across metallicity, the Gaussian mean of the blue GCs is expected to be brighter than that of the red one (Ashman et al. 1995; Di Criscienzo et al. 2006; Jordán et al. 2007). Observationally, our result confirms previous evidence obtained from data with much smaller surface coverage (Whitmore et al. 1995; Puzia et al. 1999; Peng et al. 2009). Additional improvements on this will be enabled by analyzing new galaxies in the VEGAS-SSS sample, with the possible inclusion of $u$ - and $r$-band data in the SSSs selection process.

As a final comment, we highlight that the depth, in terms of absolute magnitude, and the image quality for the other galaxies in the VEGAS sample will be similar to the one inspected here, thus we expect that using the tools presented here ${ }^{6}$, we will reliably analyze the color distributions and study GCs luminosity

6 Except for inspecting the size and shape which will hardly be possible for GCs in distant galaxies, but which can be done for the UCDs. 

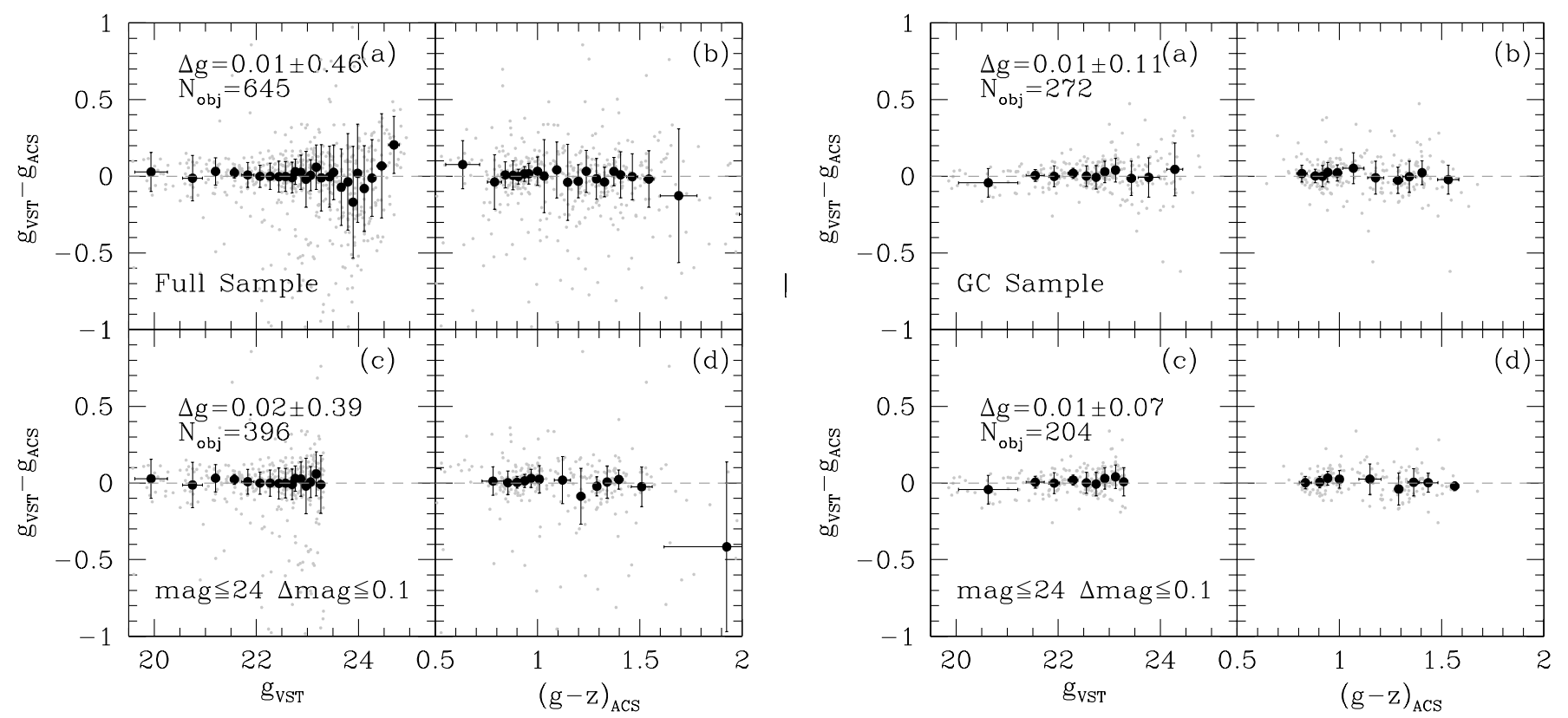

Fig. 9. Magnitude comparison between VEGAS-SSS and ACS. Left panels: a) and b) comparison for the full list of ACS to VEGAS-SSS matching sources (gray dots). The running mean and the corresponding rms are shown with black circles and error bars. The median difference, the rms and the number of objects matched are also labeled. Panels c) and d) are the same as the upper panels, but for sources with magnitude and photometric error cuts as labeled. Right: as left panels, but only GC candidates in the ACS catalog are considered.

function for all other targeted galaxies out to unreached galaxy effective radii. Moreover, for objects at larger distances the background-decontamination methods described in this section will probably be more effective because of the larger galactocentric distances inspected.

\section{Object sizes: comparison with literature and analysis}

In this section we present a detailed analysis of the properties of the SSS in the field of NGC 3115, and concentrate on the sample of objects with $R_{\mathrm{h}}$ estimates from Ishape.

\subsection{Comparing photometry and sizes derived from VEGAS-SSS and HST/ACS}

As already mentioned, using the photometry and size measurement tools described in Sect. 3.1, we finally worked with a catalog containing $~ 30000$ sources. We compared our measurements with the estimates by Jennings et al. (2014), based on HST/ACS $g_{F 475 W}$ and $z_{F 850 L P}$ observations. At the distance of the galaxy, all sources in the field of NGC 3115 with $R_{\mathrm{h}} \geq 1 \mathrm{pc}$ appear to be resolved at the pixel resolution of the ACS. Thus we assume that the $R_{\mathrm{h}}$ measured from ACS data represents the true distribution of $R_{\mathrm{h}}$ for the GCs in the galaxy within the limited common area.

The matched GC list from Jennings et al. (2014) with the VST catalog (using 0.'5 radius) contains $\sim 270$ of the 360 candidates $^{7}$. Nearly $\sim 70 \%$ of the unmatched sources are GCs located in the central galaxy regions, where we did not model and subtract the galaxy light profile. The remaining $~ 30 \%$ of missing objects are faint sources, undetected in the shallower $i$ image, or objects blended with bright neighbors. The number of missed sources beyond the central regions drops to $\sim 10$ if

\footnotetext{
7 We found a systematic shift in RA, $\triangle \mathrm{RA}(\mathrm{VST}-\mathrm{ACS}) \sim+0$.' $^{\prime}$. In our analysis we applied the correction to ACS data.
}

only the $g$-band catalog is considered (again faint or blended sources). The $g$-band ACS and VEGAS-SSS photometry agrees very well, as shown in Fig. 9 for magnitude and color. In the figure, the full ACS catalog (Jennings, priv. comm.) and the GConly sample are considered separately. For sake of homogeneity, the comparison with ACS was made using constant extinction. The large scatter for the full sample (left panels) is due to the extended background sources, whose aperture magnitude does not represent a good estimate for the total magnitude, for either ACS or VEGAS-SSS. Comparison of photometry for the GC-only (right panels in the figure) indicates a negligible mean residual difference in magnitude and color.

We also compared the $R_{\mathrm{h}}$ estimates from VEGAS-SSS with those from the ACS. In the comparison one must note that the list of SSS candidates is not contaminant-free in either catalogs, as it includes background sources whose (linear) $R_{\mathrm{h}}$ estimates are incorrect because they were derived according to the distance of the galaxy. In spite of this, the ACS VEGAS-SSS comparison is still valid since the same distance modulus is assumed in both analysis. Moreover, the size estimates are not equally good for the full sample of objects measured. Thus, we defined a reference sample of VEGAS-SSS candidates with reliable structural and photometric parameters by adopting the following criteria derived on the basis of the comparison with ACS photometry and object shape:

- Ishape $S / N \geq 30$ (Larsen 1999);

- total relative error on $R_{\mathrm{h}} \leq 30 \%$;

- for each source where the iteration to derive $R_{\mathrm{h}}$ was successful, Ishape provides a cutout of the image with the object analyzed, the model brightness profile, the residuals between them, and the weighting map (Larsen 1999). To reject sources with large residuals (see Fig. C.4), but otherwise good S/N and FWHM error, we chose a limit of median/rms $\leq 0.3$ for good candidates after various experiments where we inspected the statistical properties of the residual cutouts. This criterion applied for objects with 

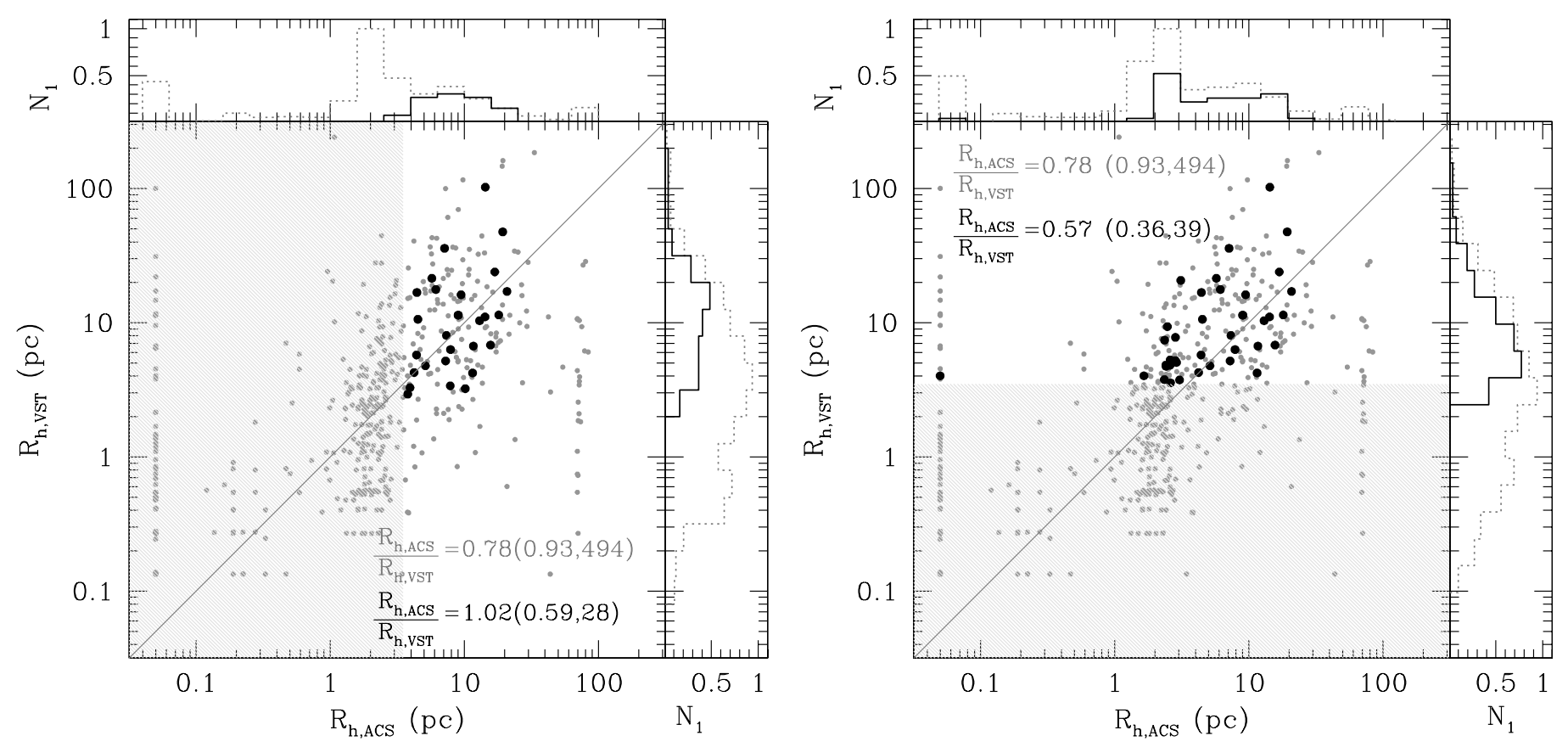

Fig. 10. Size comparison between VEGAS-SSS and ACS. Left panels: effective radii for objects common to the ACS and VEGAS-SSS catalogs (full sample with gray dots, reference sample with black circles). For each sample, the median ratio between the ACS and VEGAS-SSS $R_{\mathrm{h}}$ is reported, the rms $\mathrm{MAD}$ and the number of sources used are given in parentheses. The histograms shown in the upper and right insets use the same color coding as the central panel and are normalized to 1 at peak value for the full sample. The effective radii from ACS data are used for the selection (unshaded area). The diagonal line represents the 1:1 relation. Right panels: as left panels, but the selection is based on $R_{\mathrm{h}}$ from VEGAS-SSS

contaminating neighbors or structures not accounted for by the previous criteria;

- as in Sect. 4 we adopted a color range $0.4 \leq(g-i) \leq$ 1.25 mag both for GCs and UCDs;

- maximum photometric uncertainty $\Delta m_{g}=0.15 \mathrm{mag}$;

- axial ratio, $b / a \geq 0.3$. (van den Bergh \& Morbey 1984; Blakeslee \& Barber DeGraaff 2008; Cantiello et al. 2009).

Figure 10 shows the size comparison of VEGAS-SSS to ACS for the full and reference samples. A first evidence is the "coma"-shaped distribution of data. This behavior highlights the expected lack of accuracy of Ishape for objects with effective radii below $1 / 10$ the FWHM, that is, $R_{\mathrm{h}, \mathrm{VST}} \lesssim 3.5 \mathrm{pc}$ at the distance of the galaxy.

When only sources in the reference sample and with $R_{\mathrm{h}, \mathrm{ACS}} \geq 3.5 \mathrm{pc}$ are used (black filled circles in Fig. 10, left panels), the median ratio of ACS and VEGAS-SSS $R_{\mathrm{h}} \mathrm{S}$ is 1.02 ,

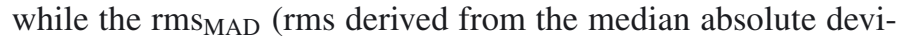
ation) of the ratio is $\sim 0.59$. Thus, for the 29 matched objects in the reference sample, the median and standard deviation of the mean are $1.02 \pm 0.11$, which provides a satisfactory agreement for the ACS and VEGAS-SSS samples when limited to the reference sample. Nevertheless, we must highlight that the $R_{\mathrm{h}}$ estimates for single objects can differ by up to a factor $\sim 5$ even for $R_{\mathrm{h}, \mathrm{ACS}} \geq 3.5 \mathrm{pc}$. More details on the differences between the size estimates for the ACS and VEGAS-SSS for extended objects are given in Appendix $\mathrm{C}$.

For the typical VEGAS target the selection will only rely on $R_{\mathrm{h}}$ measurements from VST images. The right panels of Fig. 10 show the same data as the left panels, using the $R_{\mathrm{h}, \mathrm{VST}}$ values for the selection instead of $R_{\mathrm{h}, \mathrm{ACS}}$. The comparison of ACS to VEGAS-SSS worsens, as the $R_{\mathrm{h}, \mathrm{ACS}}$ to $R_{\mathrm{h}, \mathrm{VST}}$ mean ratio and standard deviation of the mean are $0.57 \pm 0.06$. It is interesting to

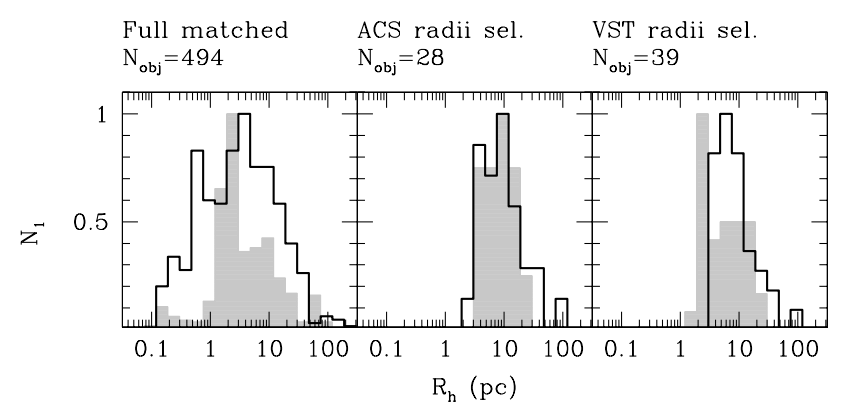

Fig. 11. $R_{\mathrm{h}}$ distribution for ACS and VEGAS-SSS samples. From left to right: $R_{\mathrm{h}}$ distribution for the full list of matched ACS and VEGASSSS sources, for the sample selected using $R_{h, \mathrm{ACS}}$, and for the sample selected using $R_{h, \mathrm{VST}}$ (see text). Shaded histograms refer to $R_{h, \mathrm{ACS}}$ distributions, solid thick lines to $R_{h, \mathrm{VST}}$. All histograms are normalized to one at peak value.

note that, taking $R_{\mathrm{h}, \mathrm{VST}}=2 \mathrm{pc}$ as lower limit, we obtain $\frac{R_{\mathrm{h}, \mathrm{ACS}}}{R_{\mathrm{h}, \mathrm{VST}}}=$ $0.78 \pm 0.08$ ( $\mathrm{rms}=0.55,46$ objects). This suggests that even though the nominal limit for Ishape is $1 / 10$ of the $F W H M$, or $\sim 3.5 \mathrm{pc}$ at the distance of NGC 3115 , the tool allows separating stars from extended sources down to $2 \mathrm{pc}$.

To inspect this matter more closely, Fig. 11 shows the $R_{\mathrm{h}}$ distributions for $a$ ) the full list of matched sources from ACS and VEGAS-SSS (left panel, ACS data in gray, VST data as a thick black line); $b$ ) objects in the reference sample selected using $R_{\mathrm{h}, \mathrm{ACS}} \geq 3.5 \mathrm{pc}$ (middle panel); and $c$ ) objects in the reference sample selected using $R_{\mathrm{h}, \mathrm{VST}} \geq 3.5 \mathrm{pc}$ (right panel). The ACS and VEGAS-SSS distributions appear to be quite similar in the middle panel (case $b$ ). The $R_{\mathrm{h}}$ distributions based on VEGAS-SSS half-light radii (right panel, case $c$ ) shows a shift, 

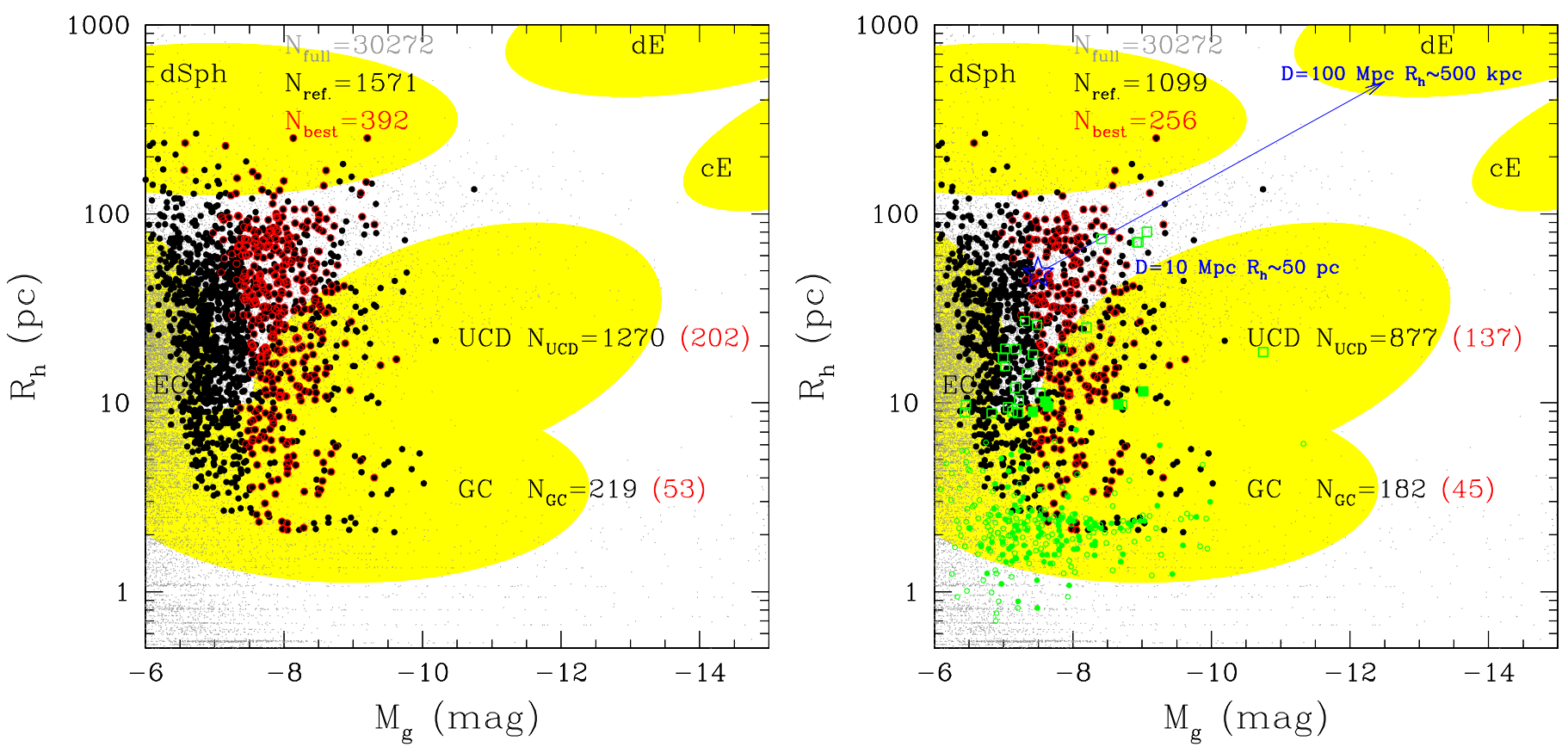

Fig. 12. Magnitude-size diagram. Left panel: magnitude versus effective radius for the VEGAS-SSS field centered on NGC 3115 . Color-coding for black and gray dots is as in previous figures, with the addition of the best sample selection (red empty circles). Yellow regions show the mean loci of the labeled SSS classes. The number of UCD and GC candidates, $N_{\mathrm{UCD}}$ and $N_{\mathrm{GC}}$, for the reference and best sample (given in parenthesis) are also reported. Right panel: as left panel, but only sources with $R_{\text {gal }} \leq R_{\mathrm{bg}}$ are plotted. The blue arrow shows the direction in which the points are shifted if the object lies at larger distance. We included the ACS sample using green symbols: GCs shown with circles, UCDs with squares. For the ACS sample, spectroscopically confirmed GCs from Arnold et al. (2011) are plotted as solid symbols.

with VEGAS-SSS radii being on average larger, and lacking the peak at $R_{\mathrm{h}} \sim 2 \mathrm{pc}$ seen in the ACS data. This behavior is due to the sources more compact than $3.5 \mathrm{pc}$, which are scattered over the entire $3.5-20 \mathrm{pc}$ interval when $R_{\mathrm{h}}$ estimates from VST are used. In particular, the list of common SSS candidates in the reference sample reaches from 28, with the selection based on the ACS radii, to 39 with the $R_{\mathrm{h}}$ selection from VST data. On this basis, we estimate that for values of $R_{\mathrm{h}} \geq 3.5$ pc the reference sample contains $\sim 30 \%$ objects with unreliable effective radii, spread across the entire $R_{\mathrm{h}}$ distribution. Of course the sample of matched objects with high-quality VEGAS-SSS sizes is relatively small (28 or 39 , depending on the selection), which makes hard to generalize the results of the comparison for the entire VST field of view.

Finally, with the aim of deriving a catalog of GC candidates from the only VST data, and estimating the contamination taking as reference the ACS GCs list, we carried out the following blind test: we adopted the selection criteria given at the beginning of this section, with the additional requirement that GC candidates must have $2 \leq R_{\mathrm{h}} \leq 8 \mathrm{pc}$ (we adopted the same $R_{\mathrm{h}}$ as used for GC by Jennings et al. 2014, for ACS data), and matched this VEGAS-SSS list with the sample of GC candidates from ACS. The results is that $\sim 20 \%$ of the candidates $(\sim 10$ over $\sim 50)$ are absent from the GC list from $\mathrm{ACS}^{8}$. In contrast, adopting as lower limit $R_{\mathrm{h}}=3.5 \mathrm{pc}$, the number of matching sources is $\sim 30$ and the contamination is nearly doubled. In other words, the test points out that the results from Ishape allow distinguishing between compact and extended sources down to $R_{\mathrm{h}}=2 \mathrm{pc}$, although the exact value of the effective radius is reliable only above $\sim 3.5 \mathrm{pc}$.

\footnotetext{
8 The result is not very sensitive to the particular choices of Ishape input parameters (see Fig. B.2).
}

In conclusion, using the reference sample obtained from the coupling of photometric and spatial extent properties, the present VEGAS-SSS catalog can be used to:

i) obtain a list of GC candidates, selected in the range $2 \leq$ $R_{\mathrm{h}}(\mathrm{pc}) \leq 8$, with an expected contamination of $\sim 20 \%$, poorly populated because of the narrow selection adopted. The number of GC candidates over the entire area inspected, $\sim 52.5^{\prime} \times 52.5^{\prime}$ ( or $\sim 145 \mathrm{kpc} \times 145 \mathrm{kpc}$ ), selected on the given photometric and size criteria is $N_{\mathrm{GC}} \sim 220$. However, for radii below $3.5 \mathrm{pc}$, the $R_{\mathrm{h}}$ are only used as an effective binary selection criterion (i.e., $R_{\mathrm{h}} \geq 2(<2)$, meaning extended (point-like) source), because this limit is smaller than the nominal limit of the tool;

ii) a catalog of extended objects with $R_{\mathrm{h}} \geq 3.5 \mathrm{pc}$, which has a contamination of $\sim 30 \%$ objects with unreliable $R_{\mathrm{h}}$ estimates.

\subsection{GC and UCD population properties based on color- and size-selection criteria}

Figure 12 shows the size-versus-magnitude diagram obtained using the reference sample, that is, with the selection criteria described in the previous section, and adopting for all objects in the field the same distance modulus. In the right panel, we plot only SSSs at galactocentric distance $R_{\text {gal }} \leq R_{\mathrm{bg}}=23^{\prime}$, corresponding to $\sim 65 \mathrm{kpc}$ at the distance of NGC 3115 . The approximate regions for GCs, UCDs, ECs, dSphs, dEs, and cEs are shown and labeled (yellow regions; mean loci are taken from Brodie et al. 2011 and Brüns \& Kroupa 2012).

Figure 12 (left panel) shows that a large fraction of selected objects in the reference sample falls within the avoidance region at $M_{g} \sim-7.5 \mathrm{mag}$ and $R_{\mathrm{h}} \gtrsim 50 \mathrm{pc}$ (Forbes et al. 2013). The situation does not seem to improve much even if only sources 


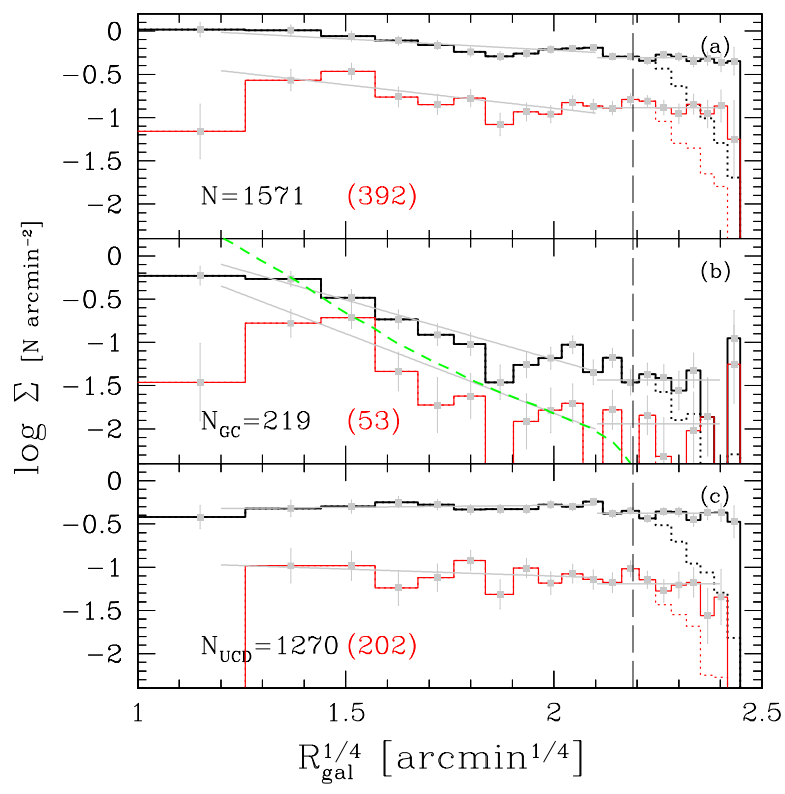

Fig. 13. Radial surface density distribution of sources in the reference (black histograms) and best (red histogram) samples. Panel a) all $R_{\mathrm{h}}$ values are taken. The $r^{1 / 4}$ fit to data is shown with gray lines. The dotted lines show the histograms without correction for areal coverage. The vertical long-dashed line is the position of limiting galactocentric radius $R_{\mathrm{bg}}$. Panel b) as upper, but only GC candidates are plotted. The green-dashed line shows the galaxy surface brightness (from Capaccioli et al., in prep., $g$ band, arbitrary scale). Panel c) as panel a), but for UCD candidates.

in the reference sample and with $S / N \geq 60$ are considered (best sample, hereafter). On the other hand, there are fewer sources in the avoidance area if only sources within $R_{\mathrm{gal}} \leq R_{\mathrm{bg}}$ are taken (Fig. 12, right panel), but these are still significantly many. Moreover, we must highlight the many UCD candidates even for the best sample and for $R_{\mathrm{gal}} \leq R_{\mathrm{bg}}$ sources $\left(N_{\mathrm{UCD}}=137\right)$.

Forbes et al. (2013) found that the avoidance zone is the result of a selection bias and confirmed the presence of various SSSs within the region, but very many sources in the avoidance area are probably background galaxies (some of them are recognizable by eye). As shown by the arrow in Fig. 12 a background source should move toward larger absolute radii and be brighter when larger distance moduli are considered.

In right panel of Fig. 12, we also plot the GC (green circles) and UCD (squares) data from Jennings et al. (2014). The spectroscopically confirmed GCs and UCDs from Arnold et al. (2011) are plotted as solid symbols. Two interesting elements here are i) the nice overlap of the overdensity region for spectroscopically confirmed GCs and VEGAS-SSS selections at $-7.5 \leq M_{g} \leq-9$ and $R_{\mathrm{h}} \sim 2-2.5 \mathrm{pc}$; and ii) the UCDs from the ACS sample outside the region where they typically occur. Three UCD candidates from the ACS sample lie at $M_{g} \sim-9$ mag and $R_{\mathrm{h}} \sim 80 \mathrm{pc}$, that is, within the zone of avoidance (if any). Two other UCDs have $M_{g} \sim-6.5 \mathrm{mag}$ and $R_{\mathrm{h}} \sim 10 \mathrm{pc}$, which typically is associated with the EC region (see also the discussion in Appendix C). This clearly shows that the distinction between the different SSS types is not trivial and sometimes contains elements of arbitrariness.

To inspect this problem in more detail, we analyzed the surface density distribution of sources versus galactocentric radius and versus $R_{\mathrm{h}}$. Figure 13 shows the radial surface density distribution for the reference and best samples, for GC candidates $\left(2 \leq R_{\mathrm{h}}(\mathrm{pc}) \leq 8\right.$, panel $\left.\mathrm{b}\right)$, and for UCD candidates

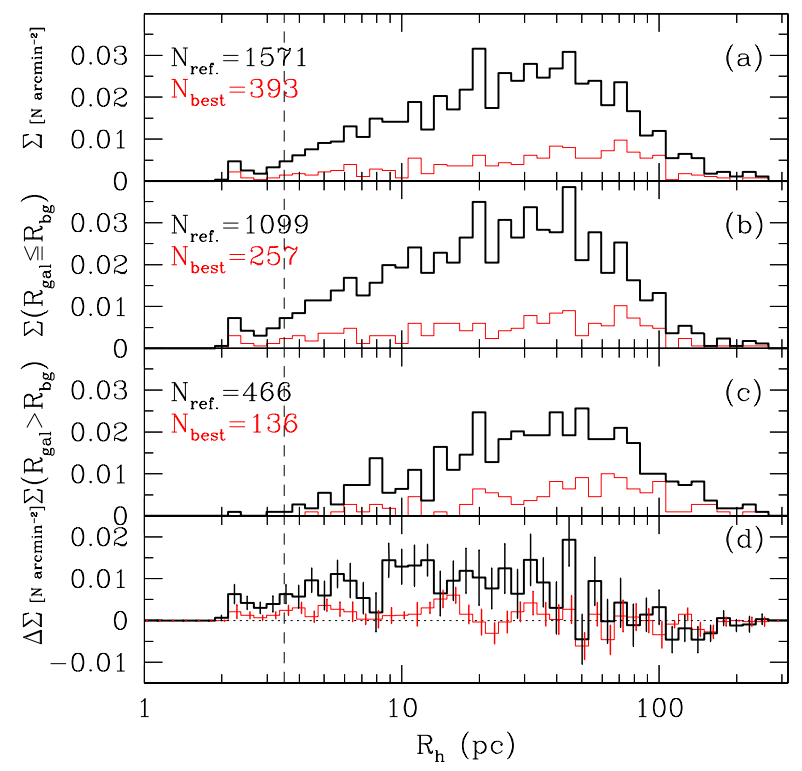

Fig. 14. $R_{\mathrm{h}}$ surface density distribution for the reference sample (black lines) and for the best sample (in red, $\Sigma$ in units of number of objects per square arcminute). The panels, from upper to lower, show the surface density over the full inspected frame for sources at $R_{\mathrm{gal}} \leq R_{\mathrm{bg}}$ for the background area at $R_{\mathrm{gal}}>R_{\mathrm{bg}}$, and for the difference between the inner and outer $23^{\prime}$. The vertical dashed line marks the $3.5 \mathrm{pc}$ limit.

$\left(8<R_{\mathrm{h}}(\mathrm{pc}) \leq 100 \text {, panel } \mathrm{c}\right)^{9}$. In each panel we also report the total number of objects selected for the reference and best sample (the latter in parentheses). The surface density for the sample with no selection on sizes (panel a) shows an obvious correlation with $R_{\text {gal }}$, and a flattening at $R_{\text {gal }} \geq R_{\text {bg }}$, suggesting that sources beyond this radius are most likely background galaxies or foreground stars. The radial density profile for GC candidates follows a de Vaucouleurs density profile out to $R_{\mathrm{bg}}$, as for the galaxy light, providing additional proof of the actual membership of the objects selected to comprise the GC population, and supporting the role of the object-size analysis carried out here. However, one must not neglect the fraction of background sources. The reference sample does not show a tendency for a radial trend in the distribution of objects with UCD-like radii. The result is expected, given the few expected UCD-candidates and the many contaminants. It is noteworthy, however, that the UCDs in the best sample show hints of a radial trend.

By integrating the fitted de Vaucouleurs $r^{1 / 4}$ density profiles from zero to $R_{\mathrm{bg}}$ for both GC and UCD density profiles after subtracting the total number of background sources we find for the reference (best) GC sample $N_{\mathrm{GC}} \sim 113(\sim 42)$ and for the best UCD sample $N_{\mathrm{UCD}} \sim 30$. The comparison of these numbers, in particular for the GCs, with the numbers in Fig. 12 (right panel), confirms our previous results that the contamination for the reference GC sample is $\sim 30 \%$, and also indicates that the best sample suffers from very little contamination. The numbers are quite different for UCDs, given the higher confusion with extended background sources. In this case, the coupling with data in other passbands will greatly reduce the contamination.

Figure 14 shows the $R_{\mathrm{h}}$ distributions for the reference and best samples, normalized to the area inspected: full detector area,

\footnotetext{
9 To estimate the density of sources, the effective area coverage in each annulus is corrected for the annular area outside the image and for the central uninspected regions (dashed histograms show the uncorrected distributions). Poisson statistics is adopted to estimate the errors.
} 
objects within the $R_{\mathrm{gal}} \leq R_{\mathrm{bg}}$ area, objects outside $R_{\mathrm{gal}}>R_{\mathrm{bg}}$, and the difference between the latter (from panel a to panel d).

The reference sample over the entire VST area (upper panel) shows a slightly increasing surface density for increasing $R_{\mathrm{h}}$ up to $\sim 50 \mathrm{pc}$, while the $R_{\mathrm{h}}$ distribution for the best sample is rather flat. The differences of the surface density for objects within $R_{\mathrm{bg}}$ or in the outside area, shown in panels b and c of Fig. 14, are quite obvious, especially for the overdensity of objects with GClike radii, $3.5 \leq R_{\mathrm{h}}(\mathrm{pc}) \leq 8$. Because of the contamination, the density of sources with GC-like radii is non-zero in the outer radius (panel c). This is in part due to the expected fraction of galaxy GCs that might lie at large galactocentric distances (see Sect. 4), while most of the contribution comes from background contamination. Indeed, the mean density of objects with $3.5 \leq$ $R_{\mathrm{h}}(\mathrm{pc}) \leq 8$ at radii $R_{\mathrm{gal}}>R_{\mathrm{bg}}$ (panel c) is $\sim 30 \%$ of the density at $R_{\text {gal }} \leq R_{\text {bg }}$ (panel b). This result confirms our previous estimate of the fraction of contamination for the color- and size-selected reference sample. Furthermore, we note that the density in the background region is $\lesssim 15 \%$ of the density in the inner regions for the best sample.

The differences between inner and outer density are better visible in panel d of Fig. 14, where we show $\Delta \Sigma \equiv \Sigma\left(R_{\text {gal }} \leq\right.$ $\left.R_{\mathrm{bg}}\right)-\Sigma\left(R_{\mathrm{gal}}>R_{\mathrm{bg}}\right)$ versus $R_{\mathrm{h}}$. Here, the surface density of sources with $R_{\mathrm{h}} \gtrsim 70 \mathrm{pc}$ is consistent with zero. More specifically, the $\Delta \Sigma$ distribution for the reference sample (black histogram) is generally consistent with zero density from $R_{\mathrm{h}} \gtrsim$ $50 \mathrm{pc}$ (with some possible candidates at $R_{\mathrm{h}} \sim 55-60 \mathrm{pc}$ ), and for $R_{\mathrm{h}} \sim 8 \mathrm{pc}$, while for the best sample various regions are compatible with zero density (e.g $R_{\mathrm{h}} \sim 7-10 \mathrm{pc}, \sim 20-30 \mathrm{pc}$, and $\geq 40 \mathrm{pc}$ ). These results imply that the surface density of objects with such $R_{\mathrm{h}}$ values is constant across the inspected area, as expected for a uniform background contamination. In other words, panel d suggests that most sources falling in the zone of avoidance (Fig. 12) are background galaxies. Second, the overabundance of sources with $R_{\mathrm{h}}$ that have GC-like radii appears clearly both for the reference and best samples. Furthermore, for the reference sample, we find a positive density of sources around the characteristic $R_{\mathrm{h}}$ values of UCDs (between 10 and $40 \mathrm{pc}$ ), which confirms the membership to this class for some of the selected objects. This overdensity, however, is weaker for the best sample, and possibly consistent with zero in some cases.

\section{Summary}

We presented the first results of the VEGAS survey for the specific science case of small stellar systems (SSSs). We described the methodology for the photometry and the size analysis of SSS candidates in the field of NGC 3115, a well-studied lenticular galaxy, and showed the potential of the survey in providing original results on SSS-related science.

The VEGAS survey will collect the deep $g$ and $i$ imaging of bright early-type galaxies with $c z \leq 4000 \mathrm{~km} \mathrm{~s}^{-1}$, possibly complemented with $r$ for most of the targets, and also with $u$-band observations for selected galaxies. One of the great advantages of VEGAS-SSS is the use of wide-field imaging, $\sim 1$ square degree, which allows studying the properties of SSSs out to very large galactocentric distances, with an accurate characterization of the background-contaminating objects. For the specific case of NGC 3115 we inspected the properties of SSSs out to $\sim 23^{\prime}$, which is more than twenty times the effective radius of the galaxy.

We first analyzed the properties of the GCs system. Because GCs are the population of SSSs numerically most abundant in the galaxy, their properties can be derived using solely photometric information, color, and magnitudes by comparing the surface density of sources in the inner galaxy regions with the density in the outer regions. Our results can be roughly divided into two groups: i) results that repeat previous analyses, giving us the opportunity to confirm the reliability of this study; ii) new results allowed by the use of the wide-field imaging. In the first group we include:

- the GCs have a bimodal $(g-i)$ distribution with peaks at $\sim 0.75$ and $\sim 1.0$ mag;

- red GCs are more centrally concentrated than blue GCs;

- as for the galaxy light, the radial density of GCs follows a de Vaucouleurs $r^{1 / 4}$ profile, but with a shallower slope;

- the turnover magnitude of the $g$-band GCLF, $M_{g}^{\mathrm{TOM}}$, coupled with the calibration from the ACSVCS survey, implies a distance modulus $\mu_{0}=29.95 \pm 0.3$ that agrees well with the literature.

These achievements support the results of previous studies, some of which were carried out with 8-10 m class telescopes, and are further complemented by the following compelling results:

- the color bimodality extends to more than $\sim 20$ galaxy effective radii;

- the blue GCs show a tendency toward bluer color at larger galactocentric radii $R_{\text {gal }}$, while red GCs seem to have a nearly constant color with $R_{\text {gal }}$;

- the galaxy light has a steeper density profile than the GCs, whether the blue or total fractions of GCs is taken into account;

- the slope of the surface density profile for red GCs at $R_{\mathrm{gal}} \geq$ $7.5^{\prime}$ matches that for the galaxy light, while a red GC overdensity appears in the inner galaxy regions;

- the ratio of blue to red clusters shows a trend with $R_{\text {gal }}$, with the fraction of blue GCs being slightly larger at larger radii;

- by separately analyzing the blue and red GCs, we find a $\Delta m_{g}^{\text {TOM }} \sim 0.2 \mathrm{mag}$, with the blue TOM being brighter;

- we do not find an obvious dependence of $M_{g}^{\mathrm{TOM}}$ with $R_{\mathrm{gal}}$.

Both the color and luminosity properties obtained are consistent with similar existing studies of the GC system in other early-type galaxies.

The bimodal GC system, with blue GCs more extended than the galaxy stellar light, and a deficiency of red GCs in the inner regions, has previously been observed in other early-type galaxies brighter than NGC 3115 and supports a scenario where blue GCs are associated with the galaxy halo, while red ones are more centrally concentrated and associated with the bulge stellar component in the galaxy. The overall observed properties might suggest that the galaxy has undergone a relatively quiescent evolution, without major star-forming events.

Adding the spatial extent of the sources to the color information gives an additional criterion for selecting SSSs, in particular GCs and UCDs. We used Ishape to determine the effective radius $R_{\mathrm{h}}$ of slightly extended objects in the field. By comparing our estimates for the objects in the reference sample with those in the literature obtained from ACS data, we found on average a satisfactory agreement. However, the $R_{\mathrm{h}}$ estimates for single objects can differ by up to a factor $\sim 5$ between ACS and VEGAS-SSS. Furthermore, the result is sensitive to the $R_{\mathrm{h}}$ estimate taken as reference (ACS or VEGAS-SSS) because of the contamination of the VEGAS-SSS sample. The various comparisons with the literature and with inner and outer galaxy regions suggest that the level of fore- and background contamination of our reference sample is $\sim 30 \%$, possibly reduced to 
one half for the (poorer) best sample. Future studies with new VEGAS-SSS $u$ - and $r$-band data will be used to constrain the properties of other, less populated classes of SSSs, such as cEs, in the field of NGC 3115 even more tightly.

In spite of the large uncertainties posed by the estimate of $R_{\mathrm{h}}$, the results obtained are encouraging, suggesting that similar analyses might be successfully carried out for the other targets in the survey, although at larger distances, studying the sizes will be limited to the most extended SSSs (UCDs, cEs), excluding the $\mathrm{GC}$ component for most of the targets beyond $\sim 10 \mathrm{Mpc}$ distance.

The results of this work on one hand confirm existing studies and support the validity of the analyses scheme developed here using data from the $2.6 \mathrm{~m}$ VST telescope. On the other hand, they provide new and independent results - especially for the GC properties out to the previously unreached galactocentric distance of $\sim 65 \mathrm{kpc}$ - showing the great potential for future applications to other VEGAS targets, in particular for the part of the sky not accessible to similar facilities.

As a final remark we highlight that at survey completion, for most of the VEGAS targets observations in at least one more passband other than $g$ and $i$ will be available. The selection of SSSs with another optical color would certainly reduce the percentage of contaminants, especially if $u$-band photometry is included. However, a contamination-free catalog based on purely optical photometry is basically unattainable. Since the coupling of optical data with just one near-IR band is very effective in reducing the fraction of contaminants to the GC and UCD populations to $\$ 5 \%$, the VEGAS-SSS catalogs will be perfectly suited to be complemented with single-band near-IR imaging (e.g., with a large-format near-IR imager like VISTA), to define the most complete and clean SSS catalogs possible, essential, for example, for future spectroscopic follow-up.

Acknowledgements. The optical imaging is collected at the VLT Survey Telescope using the Italian INAF Guaranteed Time Observations. The data reduction for this work was carried out with the computational infrastructures of the VST Center at Naples (VSTceN). We gratefully acknowledge INAF for financial support to the VSTceN. Part of this work was supported by PRIN-INAF 2011 (P.I.: G. Marconi), FIRB-MIUR 2008 (P.I.: G. Imbriani), PRIN-INAF 2011 (P.I.: A. Grado). M.P. acknowledges finical support from project FARO 2011 from the University of Naples Federico II. D.A.F. thanks the ARC for financial support via DP130100388. We are grateful to John P. Blakeslee and Zach Jennings for useful discussions related to this work. This research has made use of the NASA/IPAC Extragalactic Data-base (NED) which is operated by the Jet Propulsion Laboratory, California Institute of Technology, under contract with the National Aeronautics and Space Administration. This research has also made use of the SIMBAD database, operated at CDS, Strasbourg, France, and of the HyperLeda database (http://leda.univ-lyon1.fr).

\section{Appendix A: Completeness and the edge detection functions}

\section{A.1. Completeness correction}

The completeness function of the $g$-band images was determined by adding artificial stars to the original images and then reprocessing them as described in Sect. 3. The ratio between the number of artificial stars added and the number of stars recovered provides the completeness estimate. We added stars using a grid pattern with $\sim 20^{\prime \prime}$ increments in $x$ and $y$. Since the field is dominated by the light from NGC 3115 , the correction for magnitude completeness depends on the angular distance from the galaxy center (e.g., Cantiello et al. 2007). The radial-dependent completeness function is shown in Fig. A.1. To correct the luminosity

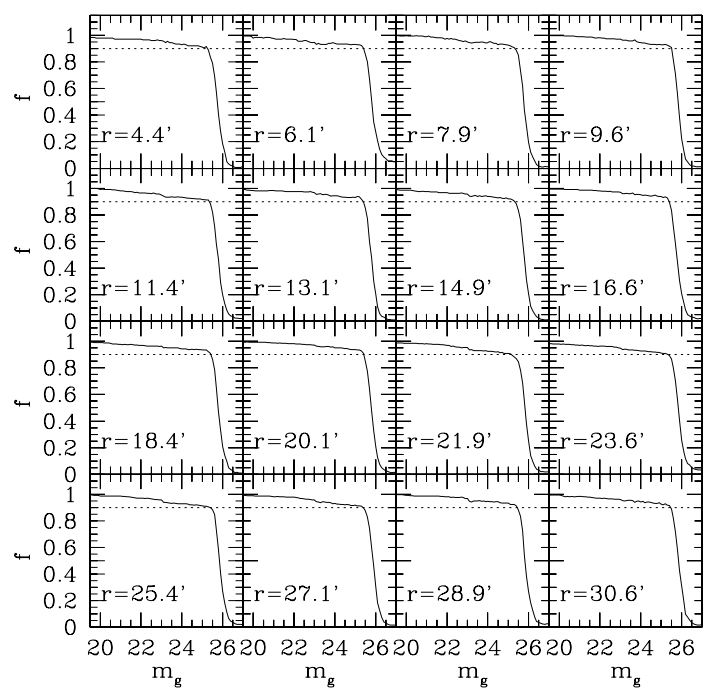

Fig. A.1. Completeness function estimated at different galactocentric radii. The label refers to the mean radius of the annulus in arcminutes. The dotted lines show the $90 \%$ completeness limit.

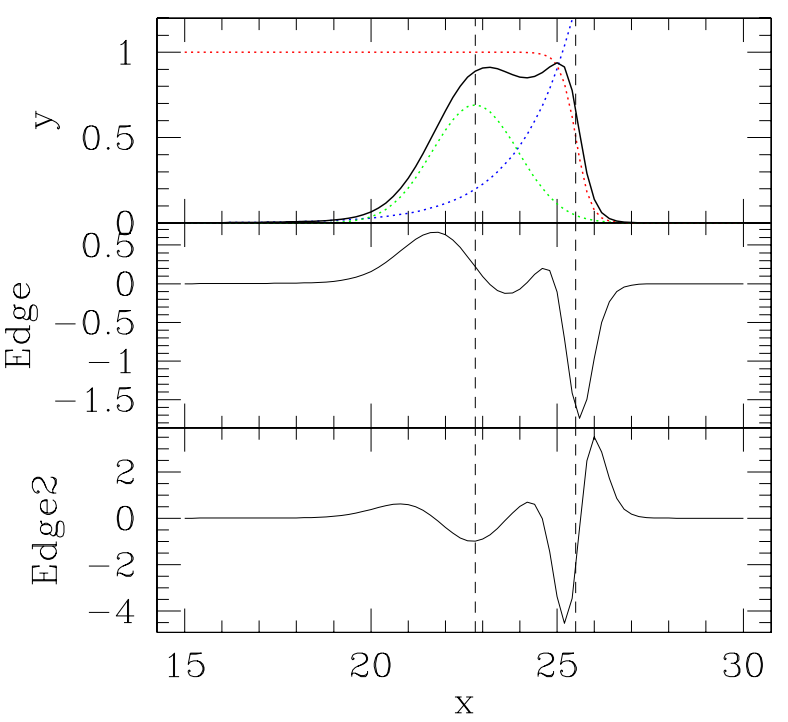

Fig. A.2. Upper panel: analytic GCLF (green line), background galaxies (blue), and completeness function (red) are combined in the total expected total luminosity function (black solid line). The GCLF peak (at $x=22.8$ ) and 50\% completeness limit (at $x=25.5$ ) are shown with dashed vertical lines. Middle panel: edge detection function applied to the analytical formula of the total luminosity function. Lower panel: second-run edge detection.

functions, the number of objects at a given magnitude is multiplied by $1 / f$ using the proper function at each galactocentric distance.

\section{A.2. Edge-detection filter}

In Fig. A. 2 we analyze the behavior of the edge-detection function on a composite function similar to the one expected for the sources in the field of NGC 3115. The function inspected is the sum of a Gaussian GCLF (Harris 2001) and a power law for background galaxies (Tyson 1988; Bernstein et al. 2002) times a completeness-like smoothed step function (green, blue, and 
Table B.1. Main Ishape parameters used for the analysis.

\begin{tabular}{lcc}
\hline \hline Parameter & Value & Explanation \\
\hline PSF & Moffat25 & Input PSF from DAOPHOT \\
\hline FITRAD & 12 & Fitting radius \\
CENTERRAD & 3 & Maximum centering radius \\
CLEANRAD & 3 & Cleaning radius \\
CTRESH & 2 & Threshold for cleaning \\
MAXCITER & 5 & Maximum number of iterations \\
CENTERMETHOD & MAX & Centering method \\
SHAPE & KING30 & Shape used for profile fitting \\
FWHMMAX & 20.0 & Maximum FWHM \\
ITMAX & 200 & Maximum number of iterations \\
ELLIPTICAL & YES & Use elliptical model \\
EPADU & 11.5 & $e-$ ADU conversion factor \\
RON & 7.0 & CCD read-out noise \\
CALCERR & YES & Calculate errors \\
\hline
\end{tabular}

red line, respectively). The edge-detection function, in a first approximation, is a derivative function and shows an inflection point at the GCLF turnover magnitude (Fig. A.2, middle panel). A second run of the edge function - edge2, roughly a second derivative - reaches a local extremum at the TOM. Thus, in first approximation, the turnover of the GCLF can be found in correspondence of an inflection and a local extremum in the edge and edge 2 functions.

\section{Appendix B: Some details on Ishape}

Ishape uses a PSF subsampled by a factor 10 relative to the resolution of the science image. To model the PSF we used the DAOPHOT package within IRAF and, to reduce the chance of contaminating the PSF modeling with GCs in the galaxy, we included in the list of PSF candidates unsaturated sources with $g-i \leq 0.3, g-i \geq 1.7$, and $m_{g} \geq 18 \mathrm{mag}$. To account for PSF variations across the image, we set DAOPHOT VARORDER $=2$, which means that the PSF is quadratically variable over the image. Then, the frame was divided into a grid of $5 \times 5$ equal subframes and the model PSF for Ishape was evaluated in the center of each subframe.

Within Ishape, we adopted the "KING30" profile, that is, the King (1962) model with concentration parameter $c=30$, which is typical for marginally resolved GCs and UCDs (Larsen \& Richtler 2000; Blakeslee \& Barber DeGraaff 2008).

To determine the best parameters for Ishape, we performed a reference run and various tests changing the input parameters. Table B.1 gives the main parameters for the reference run ( $\mathrm{g} 1$ label). The other tests were obtained as follows: for test\#1 we adopted the DAOPHOT Penny1 PSF instead of the Moffat25 (label g2); for test\#2 and \#3 (labels g3 and g4) we used Ishape fitting radius 9 pixels and 15 pixels; test\#4: the maximum FWHM is set to 40 pixels (label g5); test\#5: does not fit an elliptical model, circular symmetry is used instead (label g6). In all cases, except for test \#6, the FWHM is transformed to circularized effective radius $R_{\mathrm{h}}=1.48 \cdot F W H M_{\mathrm{KING} 30}$. $0.5 \cdot\left(1+w_{y} / w_{x}\right)$, where $F W H M_{\mathrm{KING} 30}$ and $w_{y} / w_{x}$ are the full width, and the axis ratio fitted by Ishape ${ }^{10}$. For test \#6 we used $R_{\mathrm{h}}=1.48 \cdot F W H M_{\mathrm{KING} 30}$. Figure B.1 shows the results of the Ishape tests. The data in the figure show that, in general, there

\footnotetext{
10 This equation, suggested in the Ishape handbook, provides results nearly identical to the one $R_{\mathrm{h}}=1.48 \cdot F W H M_{\mathrm{KING} 30} \cdot \sqrt{w_{y} / w_{x}}$ used by other authors (e.g., Blakeslee \& Barber DeGraaff 2008).
}

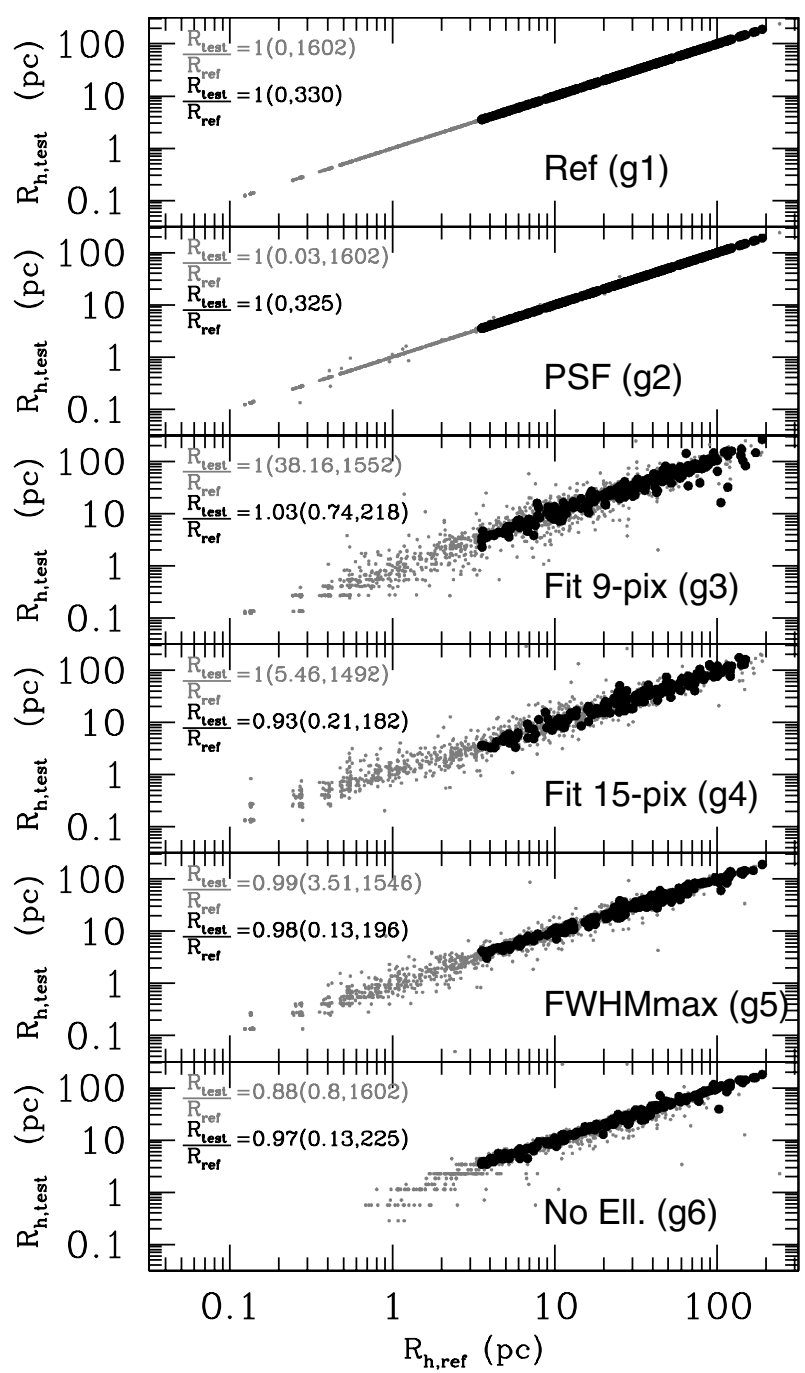

Fig. B.1. Comparison of effective radii with different Ishape input parameters. Gray circles refer to the full list of matched sources, black dots to the reference sample. The numbers in each panel show the median $R_{\mathrm{h}, \text { test }} / R_{\mathrm{h}, \text { ref }}$ ratio, with the $\mathrm{rms}_{\mathrm{MAD}}$ and the number of objects used in parenthesis, labels are color coded.

can be even a factor 10 difference between $R_{\mathrm{h}}$ estimates with different Ishape input parameters. Nevertheless, for the reference sample (see Sect. 5), the effect of changing fitting parameters implies a median change on $R_{\mathrm{h}}$ of $\lesssim 10 \%$. We also inspected the correlation between the radius and magnitude of the sources, and did not find any correlation.

Figure B. 2 shows the comparison of VEGAS-SSS to ACS for the various tests. The data in the figure show that the results with reference g1 run are broadly consistent with the other tests. We note that, in choosing the best parameters for Ishape, we also took into account the number of sources successfully analyzed by the tool. For the $g 1$ test the input catalog contained $\sim 7000$ sources, and the spatial parameters were obtained for $\sim 30000$. This number can decrease significantly for other choices of the input parameters - most notably in test g4.

\section{Appendix C: Some UCDs in Jennings et al. (2014)}

As discussed in Sect. 5 and shown in Fig. 9, we found a good match with ACS photometry. Figure C.1 shows that the 


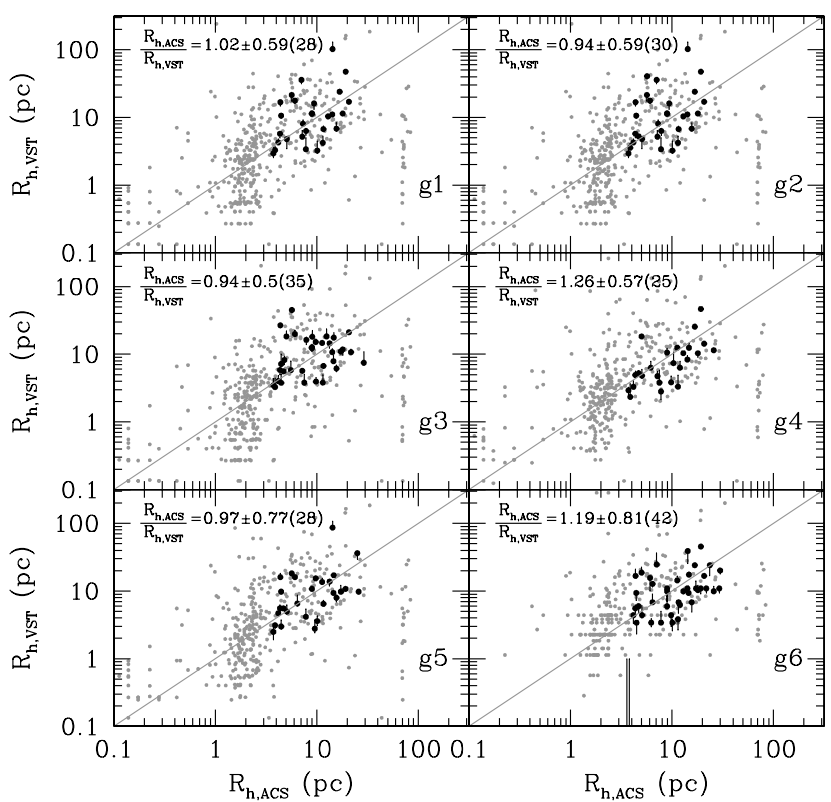

Fig. B.2. As in the left panel of Fig. 10, but for the different Ishape tests (as labeled).

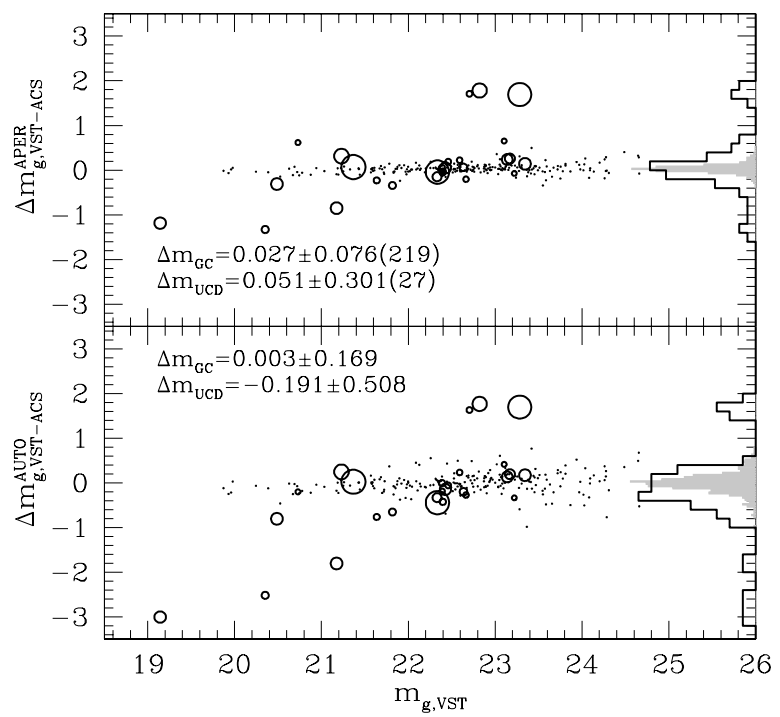

Fig. C.1. Upper panel: comparison of ACS and VEGAS-SSS aperturecorrected magnitudes for the GCs and UCDs. Dots and shaded histogram refer to GCs, UCDs are shown with empty circles (with symbol size scaled to $R_{\mathrm{h}}$ ) and thick solid line histogram. The number of matched sources is reported together with the median and rms of the VEGAS-SSS to ACS $m_{g}$ difference. Lower panel: as upper, but the SExtractor AUTO magnitude is used for both GCs and UCDs.

photometric match is not as good for some of the UCD candidates in the catalog of Jennings et al. (empty circles). The mismatch cannot be simply explained by the different aperture correction, since, as described in Jennings et al. (2014, Sect. 2.4.1), the largest aperture correction is $0.94 \mathrm{mag}$, and we find differences up to $\sim 2$ mag.

To understand the problem, we downloaded one of the ACS pointings of NGC 3115 (choosing the one with the most UCDs over the frame), and independently derived the photometry of SSSs candidates using the same methods and tools as described in Sect. 3. Figure C.2 shows the comparison VEGAS-SSS magnitudes $\left(g_{\mathrm{VST}}\right)$ with our photometry from ACS images $\left(g_{\mathrm{ACS}} t w\right)$
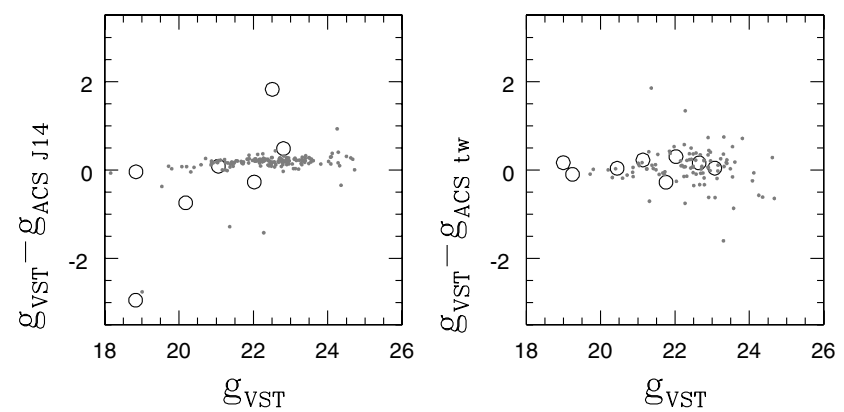

Fig. C.2. Comparison of VEGAS-SSS $g$-band photometry ( $g_{\mathrm{VST}}$ label) with our photometry of ACS data $\left(g_{\mathrm{ACS}}\right.$ tw $)$ and with measurements of Jennings et al. (2014) $\left(g_{\text {ACS } J 14}\right)$. UCDs are shown with empty circles, GCs with dots. Left panel shows the mismatch for UCDs between VEGAS-SSS and ACS measurements from Jennings et al. (2014). Right panel: as left, but our measures for the ACS pointings are used.
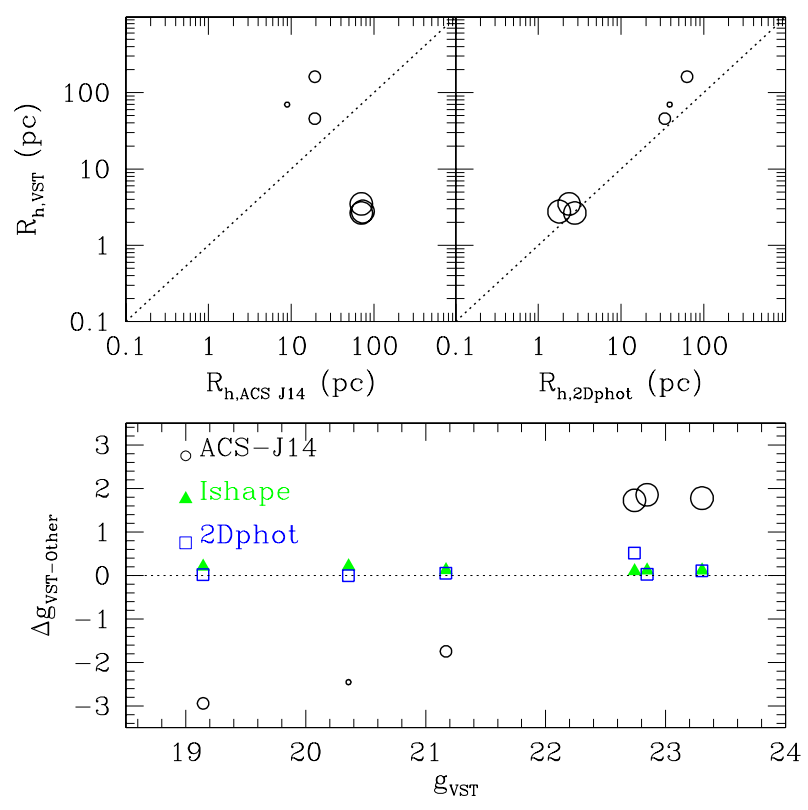

Fig. C.3. Upper panels: comparisons of effective radii for UCD candidates obtained by Jennings et al. (2014) and VEGAS-SSS results. Upper left panel: comparison between VEGAS-SSS and ACS sizes from Jennings et al. (2014). Upper right panel: comparison between the $R_{\mathrm{h}}$ from VEGAS-SSS, and measurements with 2Dphot. Lower panel: photometric comparison for the same UCDs in upper panels. VEGASSSS photometry is taken as reference. The difference with respect to ACS data from Jennings et al. (2014), magnitudes derived with Ishape, and magnitudes from 2Dphot are shown with black circles, green triangles, and blue empty squares. In all panels symbols size are scaled to the $R_{\mathrm{h}}$ from Jennings et al. (2014).

and with Jennings et al. (2014) $\left(g_{\text {ACS } J 14}\right)$. From this test, we find that, while the agreement for GC photometry is still acceptable, the large difference between ACS and VEGAS-SSS UCD data disappears (right panel in the figure). The large scatter for the GCs is mainly due to the use of only one of the ACS pointings available.

As an additional check, we also compared magnitudes and effective radii for VEGAS-SSS for the UCDs in common with the ACS pointing analyzed, using a different photometric tool, 2Dphot (La Barbera et al. 2008). We found a good match between the results of our standard procedures and those from 2Dphot (Fig. C.3, upper right and lower panels). 


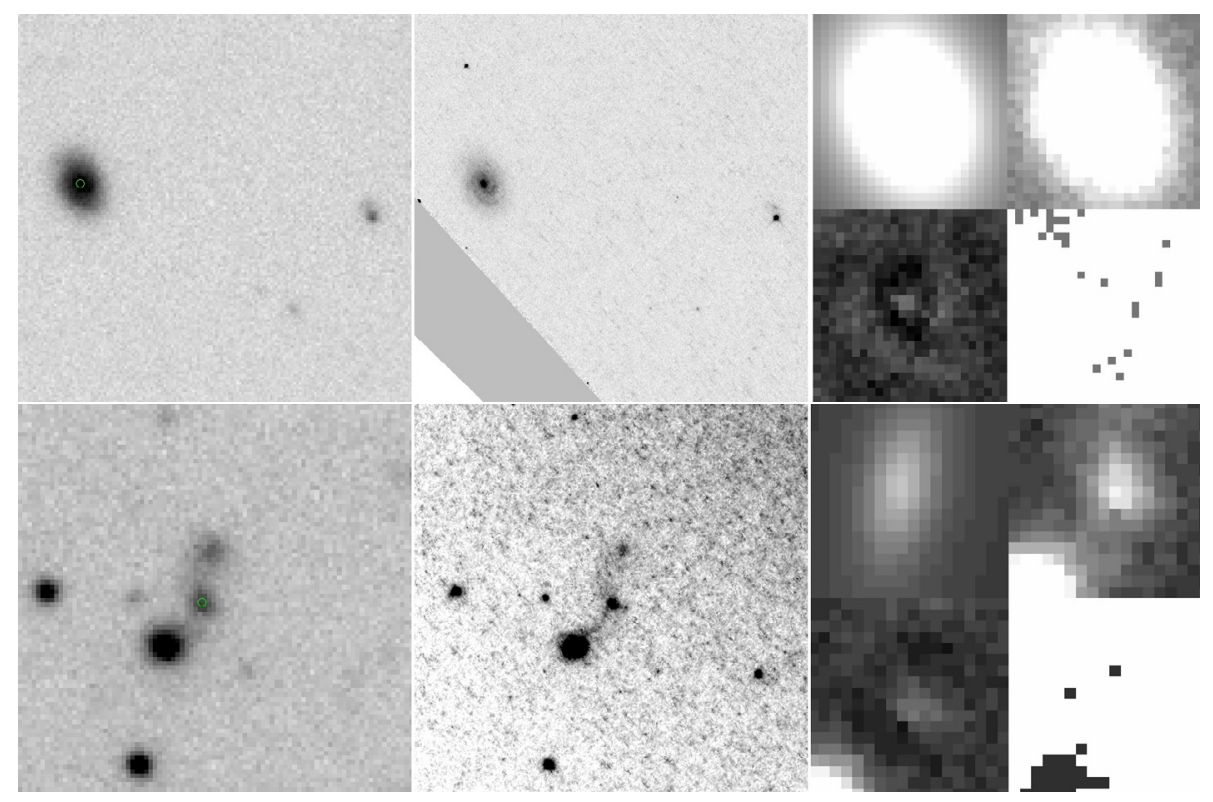

Fig. C.4. Two examples of UCD in common with Jennings et al. (2014) showing large photometric scatter with respect to VEGAS-SSS. Left and middle panels: VST and ACS $g$ image. Right panels: Ishape residual cutout. The UCD candidate is highlighted with a green circle in the VEGAS-SSS panels. Upper and lower panels refer to UCD10 and UCD20 in the catalog (15" and 10" zoom box).

A visual inspection of some UCD candidates from Jennings et al. (2014) reveals possible problems with the identification of sources. The cases shown reveal that UCD10 (the object with the largest difference in the Figs. C.1, C.2) and UCD20 from the list of Jennings et al. are actually a spiral galaxy and an object immersed in system with clear merging features (tidal streams?).

\section{References}

Alamo-Martínez, K. A., West, M. J., Blakeslee, J. P., et al. 2012, A\&A, 546, A15 Arnold, J. A., Romanowsky, A. J., Brodie, J. P., et al. 2011, ApJ, 736, L26 Ashman, K. M., \& Zepf, S. E. 1992, ApJ, 384, 50

Ashman, K. M., Conti, A., \& Zepf, S. E. 1995, AJ, 110, 1164

Bassino, L. P., Muzzio, J. C., \& Rabolli, M. 1994, ApJ, 431, 634

Bernstein, R. A., Freedman, W. L., \& Madore, B. F. 2002, ApJ, 571, 107

Bertin, E., \& Arnouts, S. 1996, A\&AS, 117, 393

Blakeslee, J. P., \& Barber DeGraaff, R. 2008, AJ, 136, 2295

Blakeslee, J. P., Cantiello, M., \& Peng, E. W. 2010, ApJ, 710, 51

Blom, C., Spitler, L. R., \& Forbes, D. A. 2012, MNRAS, 420, 37

Brodie, J. P., \& Strader, J. 2006, ARA\&A, 44, 193

Brodie, J. P., Romanowsky, A. J., Strader, J., \& Forbes, D. A. 2011, AJ, 142, 199

Brodie, J. P., Usher, C., Conroy, C., et al. 2012, ApJ, 759, L33

Brodie, J. P., Romanowsky, A. J., Strader, J., et al. 2014, ApJ, 796, 52

Brüns, R. C., \& Kroupa, P. 2012, A\&A, 547, A65

Cantiello, M., Blakeslee, J. P., Raimondo, G., et al. 2005, ApJ, 634, 239

Cantiello, M., Blakeslee, J. P., \& Raimondo, G. 2007, ApJ, 668, 209

Cantiello, M., Brocato, E., \& Blakeslee, J. P. 2009, A\&A, 503, 87

Cantiello, M., Brocato, E., \& Capaccioli, M. 2011, A\&A, 534, A35

Cantiello, M., Blakeslee, J. P., Raimondo, G., et al. 2014, A\&A, 564, L3

Capaccioli, M., \& Schipani, P. 2011, The Messenger, 146, 2

Capaccioli, M., Held, E. V., \& Nieto, J.-L. 1987, AJ, 94, 1519

Caso, J. P., Bassino, L. P., Richtler, T., Smith Castelli, A. V., \& Faifer, F. R. 2013, MNRAS, 430, 1088

Chiboucas, K., Tully, R. B., Marzke, R. O., et al. 2011, ApJ, 737, 86

Côté, P., Marzke, R. O., \& West, M. J. 1998, ApJ, 501, 554

D'Abrusco, R., Fabbiano, G., Strader, J., et al. 2013, ApJ, 773, 87

D'Abrusco, R., Fabbiano, G., Mineo, S., et al. 2014, ApJ, 783, 18

Di Criscienzo, M., Caputo, F., Marconi, M., \& Musella, I. 2006, MNRAS, 365, 1357

Dirsch, B., Richtler, T., Geisler, D., et al. 2003, AJ, 125, 1908

Dirsch, B., Schuberth, Y., \& Richtler, T. 2005, A\&A, 433, 43

Drinkwater, M. J., Gregg, M. D., Couch, W. J., et al. 2004, PASA, 21, 375

Duc, P.-A. 2014, in Galaxy Masses as Constrains of Formation Models, eds. M.

Cappelari, \& S. Courteau (Cambridge: Cambridge Univ. Press), IAU Symp., 311

A14, page 18 of 19
Durrell, P. R., Côté, P., Peng, E. W., et al. 2014, ApJ, 794, 103

Faifer, F. R., Forte, J. C., Norris, M. A., et al. 2011, MNRAS, 416, 155

Ferrarese, L., Côté, P., Cuillandre, J.-C., et al. 2012, ApJS, 200, 4

Forbes, D. A., Brodie, J. P., \& Grillmair, C. J. 1997, AJ, 113, 1652

Forbes, D. A., Sánchez-Blázquez, P., Phan, A. T. T., et al. 2006, MNRAS, 366, 1230

Forbes, D. A., Spitler, L. R., Strader, J., et al. 2011, MNRAS, 413, 2943

Forbes, D. A., Pota, V., Usher, C., et al. 2013, MNRAS, 435, L6

Forte, J. C., Faifer, F., \& Geisler, D. 2005, MNRAS, 357, 56

Galleti, S., Federici, L., Bellazzini, M., Fusi Pecci, F., \& Macrina, S. 2004, A\&A, 416, 917

Goudfrooij, P., Schweizer, F., Gilmore, D., \& Whitmore, B. C. 2007, AJ, 133, 2737

Grado, A., Capaccioli, M., Limatola, L., \& Getman, F. 2012, Mem. Soc. Astron. It. Suppl., 19, 362

Hanes, D. A., \& Harris, W. E. 1986, ApJ, 304, 599

Harris, W. E. 1991, ARA\&A, 29, 543

Harris, W. E. 1996, AJ, 112, 1487

Harris, W. E. 2001, in Saas-Fee Advanced Course 28, Star Clusters (Springer), 223

Harris, W. E., \& van den Bergh, S. 1981, AJ, 86, 1627

Harris, W. E., Kavelaars, J. J., Hanes, D. A., Hesser, J. E., \& Pritchet, C. J. 2000, ApJ, 533, 137

Huxor, A. P., Tanvir, N. R., Irwin, M. J., et al. 2005, MNRAS, 360, 1007

Huxor, A. P., Mackey, A. D., Ferguson, A. M. N., et al. 2014, MNRAS, 442, 2165

Jedrzejewski, R. I. 1987, MNRAS, 226, 747

Jennings, Z. G., Strader, J., Romanowsky, A. J., et al. 2014, AJ, 148, 32

Jordán, A. 2004, ApJ, 613, L117

Jordán, A., McLaughlin, D. E., Côté, P., et al. 2007, ApJS, 171, 101

Jordán, A., Peng, E. W., Blakeslee, J. P., et al. 2009, ApJS, 180, 54

Karachentsev, I. D., Sharina, M. E., Dolphin, A. E., et al. 2001, A\&A, 375, 359

Kartha, S. S., Forbes, D. A., Spitler, L. R., et al. 2014, MNRAS, 437, 273

King, I. 1962, AJ, 67, 471

Kissler-Patig, M. 1997, A\&A, 319, 83

Kuijken, K. 2011, The Messenger, 146, 8

Kundu, A., \& Whitmore, B. C. 1998, AJ, 116, 2841

La Barbera, F., de Carvalho, R. R., Kohl-Moreira, J. L., et al. 2008, PASP, 120, 681

Larsen, S. S. 1999, A\&AS, 139, 393

Larsen, S. S., \& Brodie, J. P. 2000, AJ, 120, 2938

Larsen, S. S., \& Brodie, J. P. 2003, ApJ, 593, 340

Larsen, S. S., \& Richtler, T. 2000, A\&A, 354, 836

Lee, M. G. 1993, ApJ, 408, 409

Liu, C., Peng, E. W., Jordán, A., et al. 2011, ApJ, 728, 116

Madrid, J. P. 2011, ApJ, 737, L13 
McConnachie, A. W. 2012, AJ, 144, 4

Mieske, S., Hilker, M., Infante, L., \& Jordán, A. 2006, AJ, 131, 2442

Mieske, S., Hilker, M., \& Misgeld, I. 2012, A\&A, 537, A3

Misgeld, I., \& Hilker, M. 2011, MNRAS, 414, 3699

Misgeld, I., Hilker, M., \& Mieske, S. 2009, A\&A, 496, 683

Muñoz, R. P., Puzia, T. H., Lançon, A., et al. 2014, ApJS, 210, 4

Muratov, A. L., \& Gnedin, O. Y. 2010, ApJ, 718, 1266

Norris, M. A., Sharples, R. M., \& Kuntschner, H. 2006, MNRAS, 367, 815

Norris, M. A., Gebhardt, K., Sharples, R. M., et al. 2012, MNRAS, 421, 1485

Peng, E. W., Ford, H. C., Freeman, K. C., \& White, R. L. 2002, AJ, 124, 3144

Peng, E. W., Côté, P., Jordán, A., et al. 2006, ApJ, 639, 838

Peng, E. W., Jordán, A., Blakeslee, J. P., et al. 2009, ApJ, 703, 42

Puzia, T. H., \& Sharina, M. E. 2008, ApJ, 674, 909

Puzia, T. H., Kissler-Patig, M., Brodie, J. P., \& Huchra, J. P. 1999, AJ, 118, 2734

Puzia, T. H., Kissler-Patig, M., Brodie, J. P., \& Schroder, L. L. 2000, AJ, 120, 777

Puzia, T. H., Zepf, S. E., Kissler-Patig, M., et al. 2002, A\&A, 391, 453
Puzia, T. H., Paolillo, M., Goudfrooij, P., et al. 2014, ApJ, 786, 78

Rejkuba, M., da Costa, G. S., Jerjen, H., Zoccali, M., \& Binggeli, B. 2006, A\&A, 448, 983

Schlafly, E. F., \& Finkbeiner, D. P. 2011, ApJ, 737, 103

Schlegel, D. J., Finkbeiner, D. P., \& Davis, M. 1998, ApJ, 500, 525

Tonini, C. 2013, ApJ, 762, 39

Tonry, J. L., Dressler, A., Blakeslee, J. P., et al. 2001, ApJ, 546, 681

Tyson, J. A. 1988, AJ, 96, 1

Usher, C., Forbes, D. A., Brodie, J. P., et al. 2012, MNRAS, 426, 1475

van den Bergh, S., \& Morbey, C. L. 1984, ApJ, 283, 598

Vanderbeke, J., West, M. J., De Propris, R., et al. 2014, MNRAS, 437, 1725

Vesperini, E. 2001, MNRAS, 322, 247

West, M. J., Cote, P., Jones, C., Forman, W., \& Marzke, R. O. 1995, ApJ, 453, L77

Whitmore, B. C., Sparks, W. B., Lucas, R. A., Macchetto, F. D., \& Biretta, J. A. 1995, ApJ, 454, L73

Yoon, S.-J., Lee, S.-Y., Blakeslee, J. P., et al. 2011, ApJ, 743, 150 